\title{
HIGHEST WEIGHT MODULES OVER GRADED LIE ALGEBRAS: RESOLUTIONS, FILTRATIONS AND CHARACTER FORMULAS
}

BY

\author{
ALVANY ROCHA-CARIDI AND NOLAN R. WALLACH ${ }^{1}$
}

\begin{abstract}
In this paper the study of multiplicities in Verma modules for Kac-Moody algebras is initiated. Our analysis comprises the case when the integral root system is Euclidean of rank two. Complete results are given in the case of rank two, Kac-Moody algebras, affirming the Kazhdan-Lusztig conjectures for the case of infinite dihedral Coxeter groups.

The main tools in this paper are the resolutions of standard modules given in [21] and a generalization to the case of Kac-Moody Lie algebras of Jantzen's character sum formula for a quotient of two Verma modules (one of the main results of this article).

Finally, a precise analogy is drawn between the rank two, Kac-Moody algebras and the Witt algebra (the Lie algebra of vector fields on the circle).
\end{abstract}

Introduction. The purpose of this paper is to begin the study of irreducible, nonstandard, highest weight modules for generalized Cartan matrix (GCM) or Kac-Moody Lie algebras. As in the case of (finite dimensional) semisimple Lie algebras the first step is to study (as Jantzen did in [10]) the case when the integral root system is of rank 2. (We will explain the concept of integral root system in detail later.)

In this paper we carry out Jantzen's program for the case of integral root systems of rank 2 in the opposite order to that of Jantzen. Jantzen showed how one can use the analysis of the integral root system of rank 2 case to derive a character formula for a quotient of two Verma modules. We derive the analogue of Jantzen's formula a priori and use it to derive the generalizations of Jantzen's results. We should point out that in the finite dimensional case our proof is quite a bit simpler than that of Jantzen.

For each of the irreducible modules for which our analysis applies, we derive (in the course of the analysis) a resolution of the same type as the Bernstein-GelfandGelfand (BGG) resolution of a finite dimensional module. (For the case of standard modules this was done in [21].)

As we have observed in [20], the highest weight modules over the Witt algebra (actually its central extension) behave as if the algebra is a rank 2, Kac-Moody Lie algebra. In the last section we make this analogy precise. In particular we derive a resolution, conjectured by V. G. Kac (see also [13]), of the trivial module by Verma

Received by the editors January 13, 1982.

1980 Mathematics Subject Classification. Primary 17B10, 17B65, 17B70; Secondary 22E65, 22 E47.

${ }^{1}$ Research partially supported by N.S.F. Grant. 
modules using (as he does in [13]) results of Goncharova [5 and 6]. We should point out that the explicit form of the homomorphisms in the exact sequence is the most important (to us) part of Theorem 6.7. This aspect is not mentioned in [13]. In the sequel to this paper [22] we will prove the full analogue of the rank 2 results in $\S 4$ for the Witt algebra (i.e. we will prove that 6.14 (iii) is true). In particular, these results imply Kac's conjectured character formulae for the Witt algebra.

In $\$ 1$ we recall the homological algebra machinery developed in [21] for infinite dimensional graded Lie algebras. In particular, our interpretation of $\operatorname{Ext}^{p}$ as the $p$ th Lie algebra cohomology of a subalgebra and the vanishing theorems on $\operatorname{Ext}^{p}$ are recalled. Using these results, we obtain information on the structure of modules from the knowledge of the 0th homology of a subalgebra (Corollary 1.7). This information is used in the derivation of the resolution of the trivial module over the Witt algebra in $\S 6$.

In $\$ 2$ we assemble the main module theoretical results of [21] on symmetrizable Kac-Moody Lie algebras. One of these is Theorem 2.4 which describes the embeddings of Verma modules by the Bruhat order in the Weyl group. It generalizes a theorem of BGG. The other is Theorem 2.5 which contains the above mentioned resolution of a standard module by direct sums of Verma modules. The uniqueness of our resolution recalled in Scholium 2.6 will be particularly important to our construction.

In $\S 3$ we obtain a generalization to symmetrizable GCM Lie algebras (of arbitrary rank) of Jantzen's character formula corresponding to the quotient of 2 Verma modules. This formula is of crucial importance in the study of characters of irreducible highest weight modules as demonstrated in the semisimple case by Jantzen's work [10]. Here it will serve as a major tool in the construction of our resolutions in the integral root system of rank 2 case. In the process we obtain elements giving embeddings of Verma modules, generalizing the constructions of Shapovalov [23] and Goodman and Wallach [7]. In the construction of a Shapovalov element we use Theorem 2.4 instead of Verma's theorem. This construction contained in Theorem 3.3 refines Kac-Kazhdan's generalization of the Shapovalov determinant formula [14].

In $\$ 4$ we construct resolutions of irreducible highest weight modules over rank 2 symmetrizable GCM's, where the highest weights lie in the Weyl group orbits of dominant integral weights. In the semisimple case these are all the integral weights. The resolutions are obtained in Theorem 4.12. The construction gives rise to filtrations of Verma modules by sums of Verma submodules. Using Theorem 4.12 we show that these filtrations coincide with Jantzen's filtration (Theorem 4.16(i)). This gives a direct description of the latter and proves Jantzen's conjecture on the heredity of his filtrations in our case. We also describe the intermediate quotients of Jantzen's filtrations (Theorem 4.16(ii)). Hence these results generalize Jantzen's results $[10,3.17,5.5]$ in the integral case. An Euler-Poincaré principle as in Garland and Lepowsky [4] gives the character of these irreducible modules (Corollary 4.13). This proves the Kazhdan-Lusztig conjectures [15] in our case.

In $\$ 5$ we handle the nonintegral case. To this end we extend the definition of integral subroot system of a weight to the case of GCM Lie algebras. Here, we 
obtain all the results obtained in $\$ 4$ for GCM Lie algebras with certain integral root systems of rank 2. For semisimple Lie algebras the results of this section contain those of $\$ 4$ including the singular case. In particular we obtain the results of Jantzen mentioned above for arbitrary highest weights with integral root systems of rank 2 . We also show that for the other GCM Lie algebras of rank 2 the results of this section almost complement the ones of $\$ 4$ for integral weights. In the Euclidean case, the analysis of $\$ \S 4$ and 5 leaves as the only outstanding case that of integral weights perpendicular to the principal null root.

The problems studied in $\S \S 4$ and 5 have strikingly similar counterparts in the case of the Witt algebra. We obtain in $\$ 6$ a resolution of the only "standard" module over the Witt algebra, namely the trivial one (Theorem 6.7). We also prove the equivalence of the analogues of the various questions answered in the analysis carried out in $\$ \S 4$ and 5 for GCM Lie algebras (Theorem 6.14). One of these questions is the irreducibility of certain modules that are constructed here. In the sequel to this paper [22] this and hence all the other questions are answered affirmatively.

We wish to thank J. Lepowsky for helpful conversations.

1. Homological algebra. We recall the definition of the pair $(\mathfrak{g}, \mathfrak{h})$ introduced in [21], where $g$ is a complex Lie algebra and $\mathfrak{h} \subset g$ is a subalgebra. We fix $\left\{\alpha_{1}, \ldots, \alpha_{n}\right\}$ $\subset \mathfrak{h}^{*}$ and denote by $Q$ the free abelian group generated by $\left\{\alpha_{1}, \ldots, \alpha_{n}\right\}$. The pair $(\mathfrak{g}, \mathfrak{h})$ has a grading: $\mathfrak{g}=\bigoplus_{\alpha \in Q} \mathfrak{g}_{\alpha}$, where $\mathfrak{g}_{\alpha}=\{X \in \mathfrak{g} \mid[H, X]=\alpha(H) X$, for all $H \in \mathfrak{h}\}, \operatorname{dim} \mathfrak{g}_{\alpha}<\infty$ for all $\alpha \in Q, \mathfrak{g}_{0}=\mathfrak{h}$ and $\left[\mathfrak{g}_{\alpha}, \mathfrak{g}_{\beta}\right] \subset \mathfrak{g}_{\alpha+\beta}, \alpha, \beta \in Q$, and satisfies the conditions (T1) and (T2) below:

(T1) $g_{\alpha} \neq(0)$ only if $\alpha=\sum_{i=1}^{n} m_{i} \alpha_{i}$ with $m_{i} \in \mathbf{Z}_{+}$for all $i$, or $-m_{i} \in \mathbf{Z}_{+}$for all $i$. (Here $\mathbf{Z}_{+}$denotes the set of all nonnegative integers.)

Let $Q^{+}=\sum_{i=1}^{n} \mathbf{Z}_{+} \alpha_{i}$, and set $\mathfrak{n}=\bigoplus_{\alpha \in Q^{+} \backslash\{0\}} \mathfrak{g}_{\alpha}$ and $\mathfrak{n}^{-}=\bigoplus_{\alpha \in Q^{+} \backslash\{0\}} \mathfrak{g}_{-\alpha}$.

(T2) There is an $\mathfrak{h}$-invariant, nondegenerate pairing between the spaces $\mathfrak{n}$ and $\mathfrak{n}^{-}$.

Examples of the above class of graded Lie algebras were given in [21]. In $\$ 6$ we consider an additional example to the ones treated in [21]. If a is a Lie algebra we denote by $U(\mathfrak{a})$ its universal enveloping algebra.

Next, we recall the definition of the truncated categories $\mathcal{C}(\lambda, q)$ defined in [21]. Let ${ }_{k} Q^{+}=\left\{\sum_{i=1}^{n} m_{i} \alpha_{i} \in Q^{+} \mid \sum_{i=1}^{n} m_{i}>k\right\}$, where $k \in \mathbf{Z}_{+}$. If $\mu=\sum_{i=1}^{n} m_{i} \alpha_{i}$ is an element of $Q$, we set $\mu^{+}=\sum_{i \in I} m_{i} \alpha_{i}$, where $i \in I \subset\{1, \ldots, n\}$ if and only if $m_{i} \in \mathbf{Z}_{+}$, and $\mu^{-}=\mu-\mu^{+}$. Fix $q \in \mathbf{Z}_{+}$and $\lambda \in \mathfrak{h}^{*}$. Let $\Pi=\Pi(\lambda, q)=$ $\left\{\mu \in \mathfrak{h}^{*} \mid \mu \in \lambda+Q\right.$ and $\left.(\mu-\lambda)^{+} \in Q \backslash \backslash_{q} Q^{+}\right\}$.

Definition 1.1 [21]. Let $\mathcal{C}=\mathcal{C}(\lambda, q)$ be the full subcategory of the category of $g$-modules such that:

(i) $M=\bigoplus_{\mu \in \mathfrak{h}^{*}} M_{\mu}$, where $M_{\mu}=\{v \in M \mid H v=\mu(H) v$, for all $H \in \mathfrak{h}\}$ and $\operatorname{dim} M_{\mu}<\infty$.

(ii) $M_{\mu} \neq(0)$ only if $\mu \in \Pi$.

Let $\mu \in \mathfrak{h}^{*}$ and $M$ be a $\mathfrak{g}$-module. The spaces $M_{\mu}$ defined as in Definition 1.1(i) are called weightspaces of $M$. If $M_{\mu} \neq(0)$ then $\mu$ is called a weight of $M$ and a nonzero element of $M_{\mu}$ is called a weight vector. If $M=U(\mathfrak{g}) v$, where $v$ is a nonzero vector of $M_{\mu}$ such that $\mathfrak{n} \cdot v=0$, then $M$ is called a highest weight module of highest 
weight $\mu$. If $\mu \in \Pi$ then it is clear that $M \in \mathcal{C}$. Let $b=\mathfrak{h} \oplus \mathfrak{n}$. We denote by $M(\mu)$ the Verma module associated with $\mathfrak{g}, \mathfrak{h},\left\{\alpha_{1}, \ldots, \alpha_{n}\right\}$ and $\mu$. That is, $M(\mu)$ is the left $U(\mathfrak{g})$-module $U(\mathfrak{g}) \otimes_{U(\mathfrak{b})} \mathbf{C}(\mu)$, where $\mathbf{C}(\mu)$ is the one-dimensional $\mathfrak{b}$-module with $\mathfrak{n}$ acting trivially and $\mathfrak{h}$ acting by $\mu$. We denote by $L(\mu)$ the unique irreducible quotient of $M(\mu)$. The irreducible modules of $\mathcal{C}$ are the $L(\mu)$, with $\mu \in \Pi$. If $M \in \mathcal{C}$ and $\mu \in \Pi$ we denote by $(M: L(\mu))$ the (generalized) multiplicities defined in [21, §5]. An object $M \in \mathcal{C}$ is said to have a Verma composition series (VCS) if it has a filtration $M=M^{1} \supset M^{2} \supset \cdots \supset M^{r} \supset M^{r+1}=(0)$ of submodules such that $M^{i} / M^{i+1} \simeq M\left(\mu_{i}\right), \mu_{i} \in \Pi, i=1, \ldots, r$. In this case $M / \mathfrak{n}^{-} M \simeq \bigoplus_{i=1}^{r} \mathbf{C}\left(\mu_{i}\right)$ as an $\mathfrak{h}$-module [21, Lemma 6.1]. If $\mu \in \Pi$ the number of indices $i \in\{1, \ldots, r\}$ such that $\mu=\mu_{i}$ is denoted by $(M: M(\mu))$ [21, Definition 6.2]. In [21] we gave a construction of projective modules in $C$. In particular, the following result was obtained:

THEOREM 1.2 [21]. The finitely generated indecomposable projective objects of $\mathcal{C}$ are in one-to-one correspondence with the irreducible objects of $\mathcal{C}: I(\mu) \leftrightarrow L(\mu), \mu \in \Pi$. Furthermore, every module $I(\mu)$ has a VCS, and

$$
(I(\mu): M(\nu))=(M(\nu): L(\mu)), \text { for all } \mu, \nu \in \Pi .
$$

REMARK. More general categories $\left(e^{S}(\lambda, q)\right)$ were introduced in [21] which are suitable to study modules induced from a "parabolic" subalgebra or generalized Verma modules. In [21] the results above were obtained in this more general setting.

We now recall some results on extensions and relative cohomology obtained in [21].

Let $\bigodot_{(\mathfrak{g}, \mathfrak{h})}$ denote the full subcategory of the category of $g$-modules $M$ such that $M=\bigoplus_{\mu \in \mathfrak{h}^{*}} M_{\mu}$. We denote by $\operatorname{Ext}_{(\mathfrak{g}, \mathfrak{h})}^{p}(M, N)$ the abelian group of equivalence classes of $p$-extensions of $\boldsymbol{M}$ by $N$ in $\bigodot_{(\mathfrak{g}, \mathfrak{h})}\left(p \in \mathbf{Z}_{+}\right)$.

The following is a particular case of Theorem 7.2 of [21].

TheOREM 1.3 [21]. Let $M \in \mathcal{C}_{(\mathfrak{g}, \mathfrak{h})}, \mu \in \mathfrak{h}^{*}, p \in \mathbf{N}$. $\operatorname{Ext}_{(\mathfrak{g}, \mathfrak{h})}^{p}(M(\mu), M)$ is naturally isomorphic with $\left(H^{p}(\mathfrak{n}, M)\right)_{\mu}$, the $\mu$-weightspace of the pth Lie algebra cohomology of $\mathrm{n}$ with coefficients in $M$.

We now state a slight variation of a particular case of [21, Theorem 7.5] which is proved exactly as that theorem was proved in [21].

TheOREM 1.4 [21]. Let $\mu \in \mathfrak{h}^{*}, M=\bigoplus_{i \in I} M_{i}, M_{i} \in \mathcal{C}\left(\lambda_{i}, q_{i}\right)$. Then

$$
\operatorname{Ext}_{(\mathfrak{g}, \mathfrak{h})}^{p}(M(\mu), M)
$$

is nontrivial only if there are distinct $\mu_{1}, \mu_{2}, \ldots, \mu_{p} \in \mathfrak{h}^{*}, \mu_{1} \neq \mu$, such that

$$
\left(M: L\left(\mu_{p}\right)\right)\left(M\left(\mu_{p}\right): L\left(\mu_{p-1}\right)\right) \cdots\left(M\left(\mu_{1}\right): L(\mu)\right) \neq 0 .
$$

Next we show how the study of the 0th Lie algebra homology of $\mathfrak{n}^{-}$with coefficients in $M \in \mathcal{C}$ yields results on the $\mathrm{g}$-module structure of $M$.

Proposition 1.5. Let $M=\bigoplus_{i=1}^{r} M_{i}, M_{i} \in \mathcal{C}\left(\lambda_{i}, q_{i}\right)$, and $E \subset M$ be an h-module such that $E \simeq H_{0}\left(\mathfrak{n}^{-}, M\right)$ under the natural h-module homomorphism $M \rightarrow$ $H_{0}\left(\mathfrak{n}^{-}, M\right)$. Then $M=U\left(\mathfrak{n}^{-}\right) E$. 
Proof. We order the weights $\mu_{1}, \mu_{2}, \ldots$ of $H_{0}\left(\mathfrak{n}^{-}, M\right)=M / \mathfrak{n}^{-} M$ according to the rule: $\mu_{i}<\mu_{j} \Rightarrow j<i$, where the partial order used is defined by $\chi<\psi \Leftrightarrow \psi-\chi$ $\in Q^{+}$. (It is clear that the weights of $\Pi$ can be enumerated as indicated.) We order the weights $\nu_{1}, \nu_{2}, \ldots$ of $M$ compatibly with the enumeration of the $\mu_{j}$ 's. Let $\mu_{1}=\nu_{j}$. If $j>1$, then $M_{\nu_{1}}=E_{\nu_{1}} \oplus\left(\mathfrak{n}^{-} M\right)_{\nu_{1}}=(0)$. Hence $j=1, \mu_{1}=\nu_{1}$ and $M_{\nu_{1}}=E_{\nu_{1}}$. Suppose we have shown that $M_{\nu_{k}} \subset U\left(\mathfrak{n}^{-}\right) E$ for all $k \leqslant i$.

$$
M_{\nu_{i+1}}=E_{\nu_{i+1}} \oplus\left(\mathfrak{n}^{-} M\right)_{\nu_{i+1}} \subset E_{\nu_{i+1}}+\sum_{k \leqslant i} \mathfrak{n}^{-} M_{\nu_{k}} \subset U\left(\mathfrak{n}^{-}\right) E,
$$

since $\left(\mathfrak{n}^{-} M_{\nu_{k}}\right)_{\nu_{i+1}} \neq(0)$ implies $k<i+1$, and by the induction assumption. Therefore $M=U\left(\mathfrak{n}^{-}\right) E$. Q.E.D.

Corollary 1.6. Let $M=\bigoplus_{i=1}^{r} M_{i}, M_{i} \in \mathcal{C}\left(\lambda_{i}, q_{i}\right)$. Then $M$ is finitely generated if and only if $H_{0}\left(\mathrm{n}^{-}, M\right)$ is finite dimensional.

Proof. We need only to observe that if $M \in \mathcal{C}\left(\lambda_{i}, q_{i}\right)$ then $M$ is $U(g)$-finitely generated if and only if $M$ is $U\left(n^{-}\right)$-finitely generated, by the Poincaré-Birkhoff-Witt theorem and the local $U(\mathfrak{n})$-finiteness of $M$. Q.E.D.

CoROllaRy 1.7. Let $M=\bigoplus_{i=1}^{s} M_{i}, M_{i} \in \mathcal{C}\left(\lambda_{i}, q_{i}\right)$, be a $U\left(\mathfrak{n}^{-}\right)$-torsion free object of $\bigodot_{(\mathfrak{g}, \mathfrak{h})}$. Let $M / \mathfrak{n}^{-} M=\bigoplus_{i=1}^{r} \mathbf{C} v_{i}$ where $v_{i}$ is a weight vector of weight $\nu_{i}$. We order the $\nu_{i}$ so that $\nu_{i}<\nu_{j} \Rightarrow j<i$. (This can obviously always be done.) If $\left(M\left(\nu_{i}\right): L\left(\nu_{j}\right)\right)=0$ for all $1 \leqslant i<j \leqslant r$, then we may choose $n$-invariant representatives of the $v_{i}$ in $M_{\nu_{i}}$, i.e., $M \simeq \sum_{i=1}^{r} M\left(\nu_{i}\right)$.

Proof. Set $M_{i}=\sum_{j=1}^{i} U\left(\mathrm{n}^{-}\right) v_{j}$. Then (0) $\subset M_{1} \subset \cdots \subset M_{r-1} \subset M_{r}$ and $M_{r} \simeq M$ by the proof of Proposition 1.5. We note that $M_{1} \simeq M\left(\nu_{1}\right)$. Suppose we have shown the assertion for all $v_{j}, 1 \leqslant j<i$. We note that $W_{i}=M_{i} / M_{i-1}$ is a highest weight module of highest weight $\nu \leqslant \nu_{i}$. If $\nu<\nu_{i}$ then $v_{i} \in M_{i-1}$, hence $\left(M\left(\nu_{j}\right): L\left(\nu_{i}\right)\right) \neq 0$ for some $j, 1 \leqslant j<i$, a contradiction. Hence $W_{i}$ is a highest weight module of highest weight $\nu_{i}$. We have an exact sequence

$$
0 \rightarrow M_{i-1} \stackrel{\alpha}{\rightarrow} M_{i} \stackrel{\beta}{\rightarrow} W_{i} \rightarrow 0 .
$$

The argument of the proof of Theorem 1.3 shows that $f: \mathfrak{n} \rightarrow M_{i-1}$, defined by $\alpha(f(X))=X v_{i}$ for all $X \in \mathfrak{n}$, yields an element $[f]$ of $H^{1}\left(\mathfrak{n}, M_{i-1}\right)_{\nu_{i}}$. This space is isomorphic with $\operatorname{Ext}_{(\mathfrak{g}, \mathfrak{h})}^{1}\left(M\left(\nu_{i}\right), M_{i-1}\right)$ by Theorem 1.3. Hence, if $[f] \neq 0$ then $0<\left(M_{i-1}: L\left(\nu_{i}\right)\right) \leqslant \sum_{j=1}^{i-1}\left(M\left(\nu_{j}\right): L\left(\nu_{i}\right)\right)$ by Theorem 1.4. This would imply that $\left(M\left(\nu_{j}\right): L\left(\nu_{i}\right)\right) \neq 0$ for some $j, 1 \leqslant j<i$, a contradiction. Therefore $[f]$ is trivial, i.e., $\beta\left(v_{i}\right)$ lifts to an $n$-invariant in $\left(M_{i}\right)_{\nu_{i}}$. This implies the assertion for the $v_{i}$ for all $i, 1 \leqslant i \leqslant r$. Q.E.D.

2. The resolution of a standard module over a Kac-Moody Lie algebra. In this section we consider a special example of the pairs $(g, \mathfrak{h})$ defined in $\S 1$. We assume

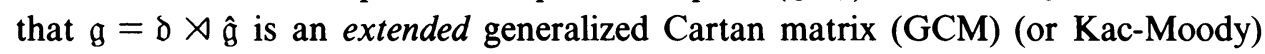
Lie algebra obtained by adjoining degree derivations to a symmetrizable GCM Lie 
algebra $\hat{g}$ over $\mathbf{C}$. Let $\mathfrak{h}=\mathfrak{d} \oplus \hat{\mathfrak{h}} \subset \mathfrak{g}$ be a (Cartan) subalgebra relative to which $\mathfrak{g}$ has a rootspace decomposition

$$
\mathfrak{g}=\mathfrak{h} \oplus \underset{\alpha \in \Delta}{\bigoplus} \mathfrak{g}_{\alpha}
$$

where $\Delta$ is the set of roots of $(\mathfrak{g}, \mathfrak{h})$. Here $\mathfrak{g}_{\alpha}=\{X \in \mathfrak{g} \mid[H, X]=\alpha(H) X$ for all $H \in \mathfrak{h}\}$. We denote by $\Delta_{+}$(resp. $\Delta_{-}$) the set of positive (resp. negative) roots. We set $\mathfrak{n}=\bigoplus_{\alpha \in \Delta_{+}} \mathfrak{g}_{\alpha}, \mathfrak{n}^{-}=\bigoplus_{\alpha \in \Delta_{-}} \mathfrak{g}_{\alpha}$ and $\mathfrak{b}=\mathfrak{h} \oplus \mathfrak{n}$. We denote by $B$ the usual symmetric bilinear $\mathfrak{g}$-invariant form on $\mathfrak{g}$. Then $\mathfrak{g}=\mathfrak{n}^{-} \oplus \mathfrak{h} \oplus \mathfrak{n}$, and $\mathfrak{g}, \mathfrak{h}, B, \mathfrak{n}$ and $\mathfrak{n}^{-}$ satisfy the conditions (T1 and T2) of $\S 1$. Let $\Delta_{0}=\left\{\alpha_{1}, \ldots, \alpha_{n}\right\}$ be the set of simple roots. Let $\left(\right.$, ) denote the symmetric bilinear form on $\mathfrak{h}^{*}$ induced by the restriction of $B$ to $\mathfrak{h}$. We define the simple reflections $\sigma_{i}=\sigma_{\alpha_{i}}$ of $\mathfrak{h}^{*}$ by

$$
\sigma_{i}(\mu)=\mu-2 \frac{\left(\mu, \alpha_{i}\right)}{\left(\alpha_{i}, \alpha_{i}\right)} \alpha_{i} \text { for all } \mu \in \mathfrak{h}^{*}, i=1, \ldots, n .
$$

Let $W$ be the Weyl group of $(\mathfrak{g}, \mathfrak{h})$, that is, $W$ is the group of automorphisms of $\mathfrak{h}^{*}$ generated by $\left\{\sigma_{i} \mid i=1, \ldots, n\right\}$. We denote by $l$ the length function on $W$ and set $W^{(k)}=\{w \in W \mid l(w)=k\}$, for all $k \in \mathbf{Z}_{+}$. Let $\Delta_{R}=\cup_{i=1}^{n} W \alpha_{i}$ denote the set of real roots and $\Delta_{I}=\Delta \backslash \Delta_{R}$ denote the set of imaginary roots. For an introduction to GCM Lie algebras the reader should consult the original papers of Kac [12] and Moody [17] (see also [16]).

If $\alpha \in \Delta_{R}$ we set $\sigma_{\alpha}(\mu)=\mu-2(\mu, \alpha) \alpha /(\alpha, \alpha)$ for all $\mu \in \mathfrak{h}^{*} . \sigma_{\alpha}$ is an element of $W$. Indeed, if $\alpha=w \alpha_{i}$ for some $w \in W$ and some $i \in\{1, \ldots, n\}$ then $\sigma_{\alpha}=w \sigma_{i} w^{-1}$ (cf. [19]).

Let $h_{1}, \ldots, h_{n}$ denote the canonical basis elements of $\hat{\mathfrak{h}}$. Let $P$ (resp. $P^{+}$) denote the set of integral (resp. dominant integral weights), i.e., $P=\left\{\mu \in \mathfrak{h}^{*} \mid \mu\left(h_{i}\right) \in \mathbf{Z}\right.$, $i=1, \ldots, n\}$ and $P^{+}=\left\{\mu \in \mathfrak{h}^{*} \mid \mu\left(h_{i}\right) \in \mathbf{Z}_{+}, i=1, \ldots, n\right\}$. Fix an element $\rho \in \mathfrak{h}^{*}$ such that $\rho\left(h_{i}\right)=1, i=1, \ldots, n$. Clearly $\rho \in P^{+}$. If $w \in W$ and $\mu \in \mathfrak{h}^{*}$ we write $w \cdot \mu=w(\mu+\rho)-\rho$.

We recall a useful result obtained in [21, Lemma 8.2]. Set $h_{\alpha_{i}}=\left(\alpha_{i}, \alpha_{i}\right) h_{i} / 2$, $i=1, \ldots, n$, and $h_{\alpha}=\sum_{i=1}^{n} k_{i} h_{\alpha_{i}}$, if $\alpha=\sum_{i=1}^{n} k_{i} \alpha_{i}$.

Lemma 2.1 [21]. Let $\mu \in P^{+}, w \in W$ and $\beta \in \Delta_{+}$. The following statements are equivalent:

(i) $2 w(\mu+\rho)\left(h_{\beta}\right)=n(\beta, \beta)$ with $n \in \mathbf{N}$;

(ii) $\beta \in \Delta_{R}$ and $l\left(\sigma_{\beta} w\right)>l(w)$.

Next, we will state structural results on Verma modules obtained in [21].

Lemma 2.2 [21]. Let $\mu \in P^{+}, w, w^{\prime} \in W$. Then $\operatorname{dim} \operatorname{Hom}_{\mathfrak{g}}\left(M\left(w^{\prime} \cdot \mu\right), M(w \cdot \mu)\right) \leqslant$ 1.

Since an element of $\operatorname{Hom}_{g}(M(\chi), M(\psi))$ is either zero or injective and because of the above lemma we write $M\left(w^{\prime} \cdot \mu\right) \subset M(w \cdot \mu)$ whenever

$$
\operatorname{Hom}_{\mathfrak{g}}\left(M\left(w^{\prime} \cdot \mu\right), M(w \cdot \mu)\right) \neq 0, \quad w, w^{\prime} \in W, \mu \in P^{+} .
$$


Next, we recall the definition of the Bruhat order on $W$. Let $\Delta_{R}^{+}=\Delta_{+} \cap \Delta_{R}$.

Definition 2.3 [21]. Let $w, w^{\prime} \in W, \alpha \in \Delta_{R}^{+}$. We write $\stackrel{\alpha}{\leftarrow} w^{\prime}$ if $w=\sigma_{\alpha} w^{\prime}$ and $l(w)=l\left(w^{\prime}\right)+1$. We write $w \leftarrow w^{\prime}$ if there is $\gamma \in \Delta_{R}^{+}$such that $w \stackrel{\gamma}{\leftarrow} w^{\prime}$. We write $w \leqslant w^{\prime}$ if $w=w^{\prime}$ or if there are $w_{0}, w_{1}, \ldots, w_{r} \in W$ such that $w=w_{0} \leftarrow w_{1} \leftarrow \cdots \leftarrow$ $w_{r}=w^{\prime}$.

Next, we state our generalization of the BGG theorem (cf. [3, Theorem 7.7.7]).

THEOREM 2.4 [21]. Let $\mu \in P^{+}, w, w^{\prime} \in W$. Then

(i) $M\left(w^{\prime} \cdot \mu\right) \subset M(w \cdot \mu) \Leftrightarrow w^{\prime} \leqslant w \Leftrightarrow\left(M(w \cdot \mu): L\left(w^{\prime} \cdot \mu\right)\right) \neq 0$.

(ii) If $M\left(w^{\prime} \cdot \mu\right) \subset M(w \cdot \mu), w \neq w^{\prime}$, then there are $w_{0}, w_{1}, \ldots, w_{r} \in W$ such that $l\left(w_{i+1}\right)=l\left(w_{i}\right)-1, i=0,1, \ldots, r-1, w_{0}=w^{\prime}, w_{r}=w$, and $M\left(w_{0} \cdot \mu\right) \subset M\left(w_{1} \cdot \mu\right)$ $\subset \cdots \subset M\left(w_{r} \cdot \mu\right)$.

(iii) If $l(w)=l\left(w^{\prime}\right)-2$ then the number of $w^{\prime \prime} \in W$ such that $M\left(w^{\prime} \cdot \mu\right) \subset$ $M\left(w^{\prime \prime} \cdot \mu\right) \subset M(w \cdot \mu), M\left(w^{\prime} \cdot \mu\right) \neq M\left(w^{\prime \prime} \cdot \mu\right) \neq M(w \cdot \mu)$, is 0 or 2 .

Next we will state a particular case of the resolution of a standard module obtained in [21].

Let $f_{i} \in \mathrm{g}_{-\alpha_{i}}$ and $e_{i} \in \mathrm{g}_{\alpha_{i}}$ be such that $f_{i}, h_{i}, e_{i}, i=1, \ldots, n$, are the canonical generators of $\hat{\mathrm{g}}$. A standard module $M$ over $\mathrm{g}$ is a highest weight module $M=U(\mathfrak{g}) v$, where $v \in M_{\mu}, \mathfrak{n} \cdot v=(0)$, such that $f_{i}^{m} \cdot v=0$ for some $m \in \mathbf{N}, i=1, \ldots, n$. The highest weight $\mu$ of the standard module $M$ lies in $P^{+}$(cf. [16]).

THEOREM 2.5 [21]. Let $M$ be a standard module with highest weight $\mu \in P^{+}$. There exists a resolution

$$
\cdots \rightarrow C_{j} \stackrel{d_{j}}{\rightarrow} C_{j-1} \rightarrow \cdots \rightarrow C_{1} \stackrel{d_{1}}{\rightarrow} C_{0} \stackrel{\eta}{\rightarrow} M \rightarrow 0
$$

where $C_{j}=\oplus M(w \cdot \mu), \quad w \in W^{(j)}, d_{j}=\bigoplus b_{w_{1}, w_{2}}^{j} i_{w_{1}, w_{2}}$ with $b_{w_{1}, w_{2}}^{j} \in\{ \pm 1\}$, and $i_{w_{1}, w_{2}}: M\left(w_{1} \cdot \mu\right) \rightarrow M\left(w_{2} \cdot \mu\right)$ are fixed unique (up to scalar) embeddings, $w_{1} \leqslant w_{2}$, $w_{1} \in W^{(j)}, w_{2} \in W^{(j-1)}$.

Scholium 2.6 [21]. Any complex

$$
\cdots \rightarrow C_{j} \stackrel{\gamma_{j}}{\rightarrow} C_{j-1} \rightarrow \cdots \rightarrow C_{1} \stackrel{\gamma_{1}}{\rightarrow} C_{0} \stackrel{\gamma_{0}}{\rightarrow} M \rightarrow 0
$$

such that $\gamma_{\left.j\right|_{M(w-\mu)}} \neq 0$ for all $w \in W^{(j)}, j \in \mathbf{N}$, is exact and equivalent to (2.1).

3. A generalization of a character formula of Jantzen. We retain the notation of $\S 2$. In this section we will derive a generalization of the character formula of Jantzen corresponding to the quotient of two Verma modules. To do this we will need to construct two families of elements in $U\left(\mathrm{n}^{-}\right)$. The first family will generalize the $\theta_{\alpha, n}$ of Shapovalov [23], the second will generalize the $S_{-n \alpha}$ of Goodman and Wallach [7]. We first sketch how the construction of Shapovalov [23] can be generalized to our case.

If $w \in W$, let $\Phi_{w}=\left\{\alpha \in \Delta_{+} \mid w^{-1} \alpha \in \Delta_{-}\right\}$.

The following result is well known (see e.g. [16, Proposition 2.8]). For the convenience of the reader we will include the simple proof. 
LEMMA 3.1 [16]. Let $w \in W^{(r)}$. Then there are $\alpha_{i_{1}}, \ldots, \alpha_{i_{r}} \in \Delta_{0}$ such that $w=\sigma_{i_{1}}$ $\cdots \sigma_{i_{r}}$ is a reduced expression and $\Phi_{w}$ consists of the $r$ distinct elements $\alpha_{i_{1}}, \sigma_{i_{1}} \alpha_{i_{2}}, \ldots, \sigma_{i_{1}}$ $\cdots \sigma_{i_{r-1}} \alpha_{i_{r}}$. Furthermore $\alpha_{i_{1}}$ can be taken to be any simple element of $\Phi_{w}$.

\section{Proof.}

$$
\left|\Phi_{w}\right| \leqslant r .
$$

We prove (1) by induction on $r$. If $r=0$ then the result is obvious. Suppose it is true for $0<r<s$ and let $w \in W^{(s)}$. It is easy to see that $\sigma_{i_{1}}\left(\Phi_{w} \backslash\left\{\alpha_{i_{1}}\right\}\right) \subset \Phi_{\sigma_{i_{1} w}}$. Since $l\left(\sigma_{i_{1}} w\right)=s-1$ we have $\left|\Phi_{w}\right| \leqslant\left|\Phi_{\sigma_{i, w}}\right|+1 \leqslant s$.

It is also clear that if $l(w) \geqslant 1$ then

$$
\Phi_{w} \text { contains some } \alpha_{i} \text {. }
$$

We prove the lemma by induction on $\left|\Phi_{w}\right|$. If $\left|\Phi_{w}\right|=0$ then $w=1$. Hence the result in this case is clear. Assume the result for $0 \leqslant\left|\Phi_{t}\right|<\left|\Phi_{w}\right|$. Let $\alpha_{i} \in \Phi_{w}$. Set $t=\sigma_{i} w$. If $\alpha \in \Phi_{w} \backslash\left\{\alpha_{i}\right\}$ then $\sigma_{i} \alpha \in \Phi_{t}$. Clearly $\alpha_{i} \notin \Phi_{t}$. But then $\sigma_{i} \alpha \in \Phi_{w}$ for $\alpha \in \Phi_{t}$. Hence $\sigma_{i} \Phi_{t}=\Phi_{w} \backslash\left\{\alpha_{i}\right\}$ and so $\left|\Phi_{t}\right|=r-1$. Hence the inductive hypothesis implies that $t=\sigma_{i_{2}} \cdots \sigma_{i_{r}}$ and $\Phi_{t}$ consists of the $r-1$ distinct elements $\alpha_{i_{2}}, \sigma_{i_{2}} \alpha_{i_{3}}, \ldots, \sigma_{i_{2}} \cdots \sigma_{i_{r-1}} \alpha_{i_{r}}$. So $\Phi_{w}$ consists of the $r$ distinct elements $\alpha_{i}, \sigma_{i} \alpha_{i_{2}}, \sigma_{i} \sigma_{i_{2}} \alpha_{i_{3}}, \ldots, \sigma_{i} \sigma_{i_{2}} \cdots \sigma_{i_{r-1}} \sigma_{i_{r}}$ as asserted. Q.E.D.

Recall that $\Delta_{R}=\cup_{i=1}^{n} W \alpha_{i}$. Thus in particular Lemma 3.1 implies that if $\alpha \in \Delta_{+}$ and there is $w \in W$ so that $w \alpha \in \Delta_{-}$then $\alpha \in \Delta_{R}^{+}$.

If $\alpha \in \Delta_{R}^{+}$then $\alpha=w \alpha_{i}$ for some $w \in W$ and some $1 \leqslant i \leqslant n$. Let $m(\alpha)$ be the minimum of the $l(w)$ for such $w$. Note that $m(\alpha)=0$ if and only if $\alpha \in \Delta_{0}$.

Let $P^{+}=\left\{\lambda \in \mathfrak{h}^{*} \mid(\lambda+\rho)\left(h_{i}\right) \in\{1,2, \ldots\}\right\}$. Set $\Gamma=\cup_{w \in W} w \cdot P^{+}$. Put $\Gamma_{i}^{+}=$ $\left\{\lambda \in \Gamma \mid(\lambda+\rho)\left(h_{i}\right)>0\right\}, \Gamma_{i}^{-}=\left\{\lambda \in \Gamma \mid(\lambda+\rho)\left(h_{i}\right)<0\right\}$.

If $\alpha \in \Delta, k \in \mathbf{N}$, let $\mathfrak{h}_{\alpha, k}^{*}=\left\{\lambda \in \mathfrak{h}^{*} \mid 2(\lambda+\rho, \alpha)=k(\alpha, \alpha)\right\}$.

LEMMA 3.2. If $\alpha \in \Delta_{R}^{+}$is not simple then there exists $j, 1 \leqslant j \leqslant n$ such that:

(i) $m\left(\sigma_{j} \alpha\right)<m(\alpha)$.

(ii) $\Gamma_{j}^{-} \cap \mathfrak{h}_{\alpha, k}^{*}$ is Zariski dense in $\mathfrak{h}_{\alpha, k}^{*}$ for all $k=1,2, \ldots$

Proof. Let $p=m(\alpha)$ and let $\alpha=w \alpha_{i}$ with $l(w)=p>0$. Let $w=\sigma_{i_{1}} \cdots \sigma_{i_{r}}$ as in Lemma 3.1. Then $\Phi_{w^{-1}}=\left\{\alpha_{i_{r}}, \sigma_{i_{r}} \alpha_{i_{r-1}}, \ldots, \sigma_{i_{r}} \cdots \sigma_{i_{2}} \alpha_{i_{1}}\right\}$. In particular if $t=\sigma_{i_{2}} \cdots \sigma_{i_{r}}$ and if $j=i_{1}$ then

$$
t^{-1} \alpha_{j} \in \Delta_{+}
$$

We also note that $\sigma_{j} \alpha=t \alpha_{i}$ and $l(t)=l(w)-1$. Thus $m\left(\sigma_{j} \alpha\right)<p$. Also $\boldsymbol{\sigma}_{j} \cdot\left(\Gamma_{j}^{-} \cap \mathfrak{h}_{\alpha, k}^{*}\right)=\Gamma_{j}^{+} \cap \mathfrak{h}_{\sigma_{j} \alpha, k}^{*}=t \cdot\left(t^{-1} \cdot \Gamma_{j}^{+} \cap \mathfrak{h}_{\alpha_{i, k}}^{*}\right)$. Thus $\Gamma_{j}^{-} \cap \mathfrak{h}_{\alpha, k}^{*}$ is Zariski dense in $\mathfrak{h}_{\alpha, k}^{*}$ if and only if $t^{-1} \cdot \Gamma_{j}^{+} \cap \mathfrak{h}_{\alpha_{i, k}}^{*}$ is Zariski dense in $\mathfrak{h}_{\alpha_{i, k}}^{*}$. But (3.1) implies that $t \cdot P^{+} \subset \Gamma_{j}^{+}$. Hence $t^{-1} \cdot \Gamma_{j}^{+} \supset P^{+}$, but then

$$
t^{-1} \cdot \Gamma_{j}^{+} \cap \mathfrak{h}_{\alpha_{i, k}}^{*} \supset P^{+} \cap \mathfrak{h}_{\alpha_{i, k}}^{*}
$$

which is clearly Zariski dense in $\mathfrak{h}_{\boldsymbol{\alpha}_{i, k}}^{*} \quad$ Q.E.D. 
TheOREM 3.3 (COMPare Shapovalov [23]). Let $\alpha \in \Delta_{R}^{+}$and let $k=1,2, \ldots$ Then there exists $\theta_{\alpha, k} \in U\left(\mathfrak{n}^{-} \oplus \mathfrak{h}\right)_{-k \alpha}$ satisfying the following conditions:

(1) $\left[e_{i}, \theta_{\alpha, k}\right] \subset U(\mathrm{~g})\left(h_{\alpha}+\rho\left(h_{\alpha}\right)-k(\alpha, \alpha) / 2\right)+U(\mathfrak{g}) \mathfrak{n}$.

(2) $\theta_{\alpha, k}=f_{1}^{k m_{1}} \cdots f_{n}^{k m_{n}}+\sum_{j} a_{j} b_{j}\left(\alpha=\sum_{i=1}^{n} m_{i} \alpha_{i}\right), a_{j} \in U_{p}\left(\mathfrak{n}^{-}\right), b_{j} \in U(\mathfrak{h})$ and $p<k \sum_{i=1}^{n} m_{i}$. Here $U_{p}\left(\mathfrak{n}^{-}\right)=U\left(\mathfrak{n}^{-}\right) \cap U_{p}(\mathfrak{g})$ and $\left(U_{p}(\mathfrak{g})\right)_{p \geqslant 0}$ is the canonical filtration of $U(\mathfrak{g})$.

The proof of this result follows the line of argument of Shapovalov [23] with the following modifications: We prove the result by induction on $m(\alpha)$ (which amounts to induction on $2(\rho, \alpha) /(\alpha, \alpha))$. We choose $\varepsilon=\alpha_{j}$ as in Lemma 3.2 if $m(\alpha)>0$. We replace the $B$ in (i'), (ii'), (iii') in $\S 3$ of [23] by $\Gamma_{j}^{-} \cap \mathfrak{h}_{\alpha, k}^{*}\left(\mathfrak{h}_{\alpha, k}^{*}=L_{\alpha, k}\right.$ in [23]). The arguments in the proof of (i'), (ii'), (iii') in [23] using [1] go through using [21, Theorem 8.15]. The rest of the argument in [23] now goes through unchanged in our case. Q.E.D.

As an immediate consequence of Theorem 3.3 we obtain the following generalization of a theorem of Verma's.

Corollary 3.4. Let $\lambda \in \mathfrak{h}^{*}, \alpha \in \Delta_{R}^{+}$and $m=2(\lambda+\rho, \alpha) /(\alpha, \alpha)$. If $m \in \mathbf{N}$ then $\operatorname{Hom}_{\mathrm{g}}(M(\lambda-m \alpha), M(\lambda)) \neq(0)$.

We now sketch the construction of $S_{-\mu}(\lambda)$ in [7]. Let $Q^{+}=\sum_{i=1}^{n} \mathbf{Z}_{+} \alpha_{i}$. The proof of the following result is identical in our case to that of Lemma 4.1, p. 226 in [7]. We first need some notation. Let $v_{\lambda}$ denote $1 \otimes 1$ in $M(\lambda)$. Let $(,)_{\lambda}$ denote the Shapovalov form on $M(\lambda)$ which we also look upon as a form on $U\left(n^{-}\right)$. Let $\chi$ : $\mathrm{n}^{-} \rightarrow C$ be linear and defined by $\chi\left(f_{i}\right)=1,1 \leqslant i \leqslant n$, and $\chi\left(\left[\mathrm{n}^{-}, \mathrm{n}^{-}\right]\right)=0$. Let $\chi$ : $U\left(n^{-}\right) \rightarrow C$ also denote the homomorphism extending $\chi$.

LEMMA 3.5. If $\mu \in Q^{+}$then there exists a rational function $\lambda \mapsto \bar{n}_{-\mu}(\lambda)$ of $\mathfrak{h}^{*}$ into $U\left(\mathrm{n}^{-}\right)_{-\mu}$ with the following properties:

(1) $\bar{n}_{0}(\lambda)=1$.

(2) $\left[e_{i}, \bar{n}_{-\mu}(\lambda)\right]=\bar{n}_{-\mu+\alpha_{i}}(\lambda)+\bar{n}_{-\mu, i}^{\prime}(\lambda)\left(h_{i}-\lambda\left(h_{i}\right)\right), \quad 1 \leqslant i \leqslant n$, where $\lambda \mapsto$ $\bar{n}_{-\mu, i}^{\prime}(\lambda)$ is a rational function into $U\left(\mathrm{n}^{-}\right)_{-\mu+\alpha_{i}}$. Also $\bar{n}_{-\mu+\alpha_{i}}$ and $\bar{n}_{-\mu, i}^{\prime}$ have singularity sets contained in that of $\bar{n}_{-\mu}$.

(3) $\left(\bar{n}, \bar{n}_{-\mu}(\lambda)\right)_{\lambda}=\chi(\bar{n})$ for $\bar{n} \in U(\bar{n})_{-\mu}$.

If $\mu=\sum m_{i} \alpha_{i} \in Q^{+} \backslash\{0\}$, let $|\mu|=\sum m_{i}$. Set $m=|\mu|$. Let $J(\mu)=\left\{\left(i_{1}, \ldots, i_{m}\right) \mid 1\right.$ $\leqslant i_{j} \leqslant n$ and $\left.\Sigma \alpha_{i_{j}}=\mu\right\}$. For $1 \leqslant k \leqslant m$ set $\mu_{I_{k}}=\sum_{j=k}^{m} \alpha_{i_{j}}=\mu-\sum_{j=1}^{k-1} \alpha_{i_{j}}$. Set $f_{I}=$ $f_{i_{1}} \cdots f_{i_{m}}$.

Define for $\lambda \in \mathfrak{h}^{*}, I \in J(\mu)$

$$
P_{I}(\lambda, \mu)=\prod_{k=1}^{m}\left(2(\lambda+\rho)-\mu_{I_{k}}, \mu_{I_{k}}\right) .
$$

If $\lambda, \mu \in \mathfrak{h}^{*}$, let $L_{\mu}(\lambda)=(2(\lambda+\rho)-\mu, \mu)$. Set $\Omega^{\prime}=\left\{\lambda \in \mathfrak{h}^{*} \mid L_{\mu}(\lambda) \neq 0\right.$ for all $\left.\mu \in Q^{+} \backslash\{0\}\right\}$.

The following result is proved in exactly the same way as Corollary 4.3, p. 229 of [7] using the Kac-Casimir operator of $\mathfrak{g}$. 
LEMMA 3.6. Let $\mu \in Q^{+} \backslash\{0\}, \mu=\sum_{i=1}^{n} m_{i} \alpha_{i}$. If $\lambda \in \Omega^{\prime}$ then

$$
\bar{n}_{-\mu}(\lambda)=\left(\prod_{i=1}^{n}\left(\alpha_{i}, \alpha_{i}\right)^{m_{i}}\right) \sum_{I \in J(\mu)} P_{I}(\lambda, \mu)^{-1} f_{I} .
$$

If $\mu \in Q^{+}$we define $r_{\mu}$ by the following formula:

$$
r_{\mu}=\prod_{\gamma} L_{\gamma}, \quad \gamma=j \alpha, \quad j>0, \quad j \alpha \leqslant \mu .
$$

Here $\nu_{1}<\nu_{2}$ if $\nu_{2}-\nu_{1} \in Q^{+}$.

Using the results of Kac and Kazhdan [14] it is easy to see that the proof of the following result is identical with the proof of Lemma 4.4, p. 231 in [7].

Lemma 3.7. Let $\mu \in Q^{+}$. Then the function $S_{-\mu}(\lambda)=r_{\mu}(\lambda) \bar{n}_{-\mu}(\lambda)$ extends to a polynomial map of $\mathfrak{h}^{*}$ into $U\left(\mathfrak{n}^{-}\right)_{-\mu}$.

We look upon $U\left(\mathfrak{n}^{-} \oplus \mathfrak{h}\right)$ as canonically equal to $U\left(\mathfrak{n}^{-}\right) \otimes U(\mathfrak{h})$. We set for $\alpha \in \Delta_{R}^{+}, k \in \mathbf{N}$

$$
\theta_{\alpha, k}(\lambda)=(1 \otimes \lambda)\left(\theta_{\alpha, k}\right)
$$

We note that

$$
\theta_{\alpha, k} v_{\lambda}=\theta_{\alpha, k}(\lambda) v_{\lambda}
$$

and

$$
\chi\left(\theta_{\alpha, k}(\lambda)\right) \equiv 1, \quad \alpha \in \Delta_{R}^{+}, k=1,2, \ldots
$$

We set $q_{\mu}(\lambda)=\chi\left(S_{-\mu}(\lambda)\right)$.

LEMMA 3.8. Let $\mu \in Q^{+}$.

(1) If $\bar{n} \in U\left(\mathfrak{n}^{-}\right)_{-\mu}$ then $\left(S_{-\mu}(\lambda), \bar{n}\right)_{\lambda}=\chi(\bar{n}) r_{\mu}(\lambda)$.

(2) $\left(S_{-\mu}(\lambda), S_{-\mu}(\lambda)\right)_{\lambda}=q_{\mu}(\lambda) r_{\mu}(\lambda)$.

This result is an obvious consequence of Lemma 3.5.

If $\alpha \in \Delta_{R}^{+}$set $\left(\mathfrak{h}_{\alpha, k}^{*}\right)^{\prime}=\left\{\lambda \in \mathfrak{h}_{\alpha, k}^{*} \mid L_{j \beta}(\lambda) \neq 0\right.$ for $\beta \in \Delta^{+}, j=1, \ldots$, and $j \beta \neq$ $k \alpha\}$. Set $V_{\alpha, k}=\left\{\lambda \in \mathfrak{h}_{\alpha, k}^{*} \mid L_{j \beta}(\lambda) \neq 0\right.$ for $\beta \in \Delta^{+}, j=1, \ldots$ and $\left.j \beta<k \alpha, j \beta \neq \kappa \alpha\right\}$.

LEMMA 3.9. Let $\alpha \in \Delta_{R}^{+}$.

(1) If $\lambda \in \mathfrak{h}_{\alpha, k}^{*}$ then $S_{-k \alpha}(\lambda)=q_{k \alpha}(\lambda) \theta_{\alpha, k}(\lambda)$.

(2) If $\lambda \in V_{\alpha, k}$ then $q_{k \alpha}(\lambda) \neq 0$.

Proof. Let $\xi \in \Omega^{\prime}$. Then Lemma 3.5 implies

$$
\left[e_{i}, S_{-k \alpha}(\xi)\right]=r_{k \alpha}(\xi)\left(\bar{n}_{-k \alpha+\alpha_{i}}(\xi)+\bar{n}_{-k \alpha, i}^{\prime}(\xi)\left(h_{i}-\xi\left(h_{i}\right)\right)\right) .
$$

Specializing to $\lambda \in \mathfrak{h}_{\alpha, k}^{*}$ and using Lemma 3.5 again this implies that $e_{i} S_{-k \alpha}(\lambda) v_{\lambda}=$ 0 .

Now it is easy to see that if $\lambda \in V_{\alpha, k}$ then $\operatorname{dim} \operatorname{Hom}_{g}(M(\lambda-k \alpha), M(\lambda))=1$. Thus for $\lambda \in V_{\alpha, k}, S_{-k \alpha}(\lambda)=c(\lambda) \theta_{\alpha, k}(\lambda)$. Applying $\chi$ we find that $c(\lambda)=q_{k \alpha}(\lambda)$ on $V_{\alpha, k}$. But $V_{\alpha, k}$ is clearly Zariski dense in $\mathfrak{h}_{\alpha, k}^{*}$. This proves (1).

To prove (2) we first assume that $\lambda \in V_{\alpha, k}$ and that $S_{-k \alpha}(\lambda)=0$. Then

$$
S_{-k \alpha}(\lambda+t \rho)=t v(t)
$$


with $v$ a polynomial in $t$. Also

$$
S_{-k \alpha}(\lambda+t \rho)=r_{k \alpha}(\lambda+t \rho) \bar{n}_{-k \alpha}(\lambda+t \rho)=t h(t) \bar{n}_{-k \alpha}(\lambda+t \rho)
$$

with $h(0) \neq 0$. Thus if $S_{-k \alpha}(\lambda)=0$ then $\bar{n}_{-k \alpha}(\lambda+t \rho)$ is defined at $t=0$. But

$$
\left(\theta_{\alpha, k}(\lambda), \bar{n}_{-k \alpha}(\lambda+t \rho)\right)_{\lambda+t \rho} \equiv 1 .
$$

Evaluating this equation at $t=0$ gives

$$
\left(\theta_{\alpha, k}(\lambda),\left.\bar{n}_{-k \alpha}(\lambda+t \rho)\right|_{t=0}\right)_{\lambda}=1 .
$$

However, by its construction $\theta_{\alpha, k}(\lambda)$ is in the radical of $(,)_{\lambda}$. Thus we have a contradiction. Hence $S_{-k \alpha}(\lambda) \neq 0$ for $\lambda \in V_{\alpha, k}$. But then $q_{k \alpha}(\lambda) \neq 0$ since $\theta_{\alpha, k}(\lambda)$ $\neq 0$ all $\lambda$. Q.E.D.

We now come to the main result of this section. Let $\alpha \in \Delta_{R}^{+}$and let $\lambda \in \mathfrak{h}_{\alpha, k}^{*}$, $k \in \mathbf{N}$. The map $X \mapsto X \theta_{\alpha, k}(\lambda) v_{\lambda}$ induces an embedding

$$
M(\lambda-k \alpha) \stackrel{T(\lambda)}{\rightarrow} M(\lambda), \quad \lambda \in \mathfrak{h}_{\alpha, k}^{*} .
$$

Set $N(\lambda)=M(\lambda) / T(\lambda) M(\lambda-k \alpha)$. Let $\omega \in \mathfrak{h}^{*}$ be such that $(\omega, \alpha)=0$ and $(\omega, \beta) \neq 0$ for $\beta \in \Delta^{+}, \beta \neq \alpha$. Then using $\omega$ and arguing as in Jantzen [10, §5], we can construct a filtration of $N(\lambda)$ by $g$-invariant subspaces.

We give, here, a slightly different derivation of the filtration which will be useful in our proof of the main result.

Fix $f_{n+1}, f_{n+2}, \ldots, \in \mathfrak{n}^{-}$so that $f_{1}, f_{2}, \ldots$ is a basis of $\mathrm{n}^{-}$by root vectors. Let $\alpha=\sum m_{i} \alpha_{i}$. Set $\bar{\theta}_{\alpha, k}=f_{1}^{k m_{1}} \cdots f_{n}^{k m_{n}}$. Let $\mu \in Q^{+}$and let $V_{-\mu}$ be a complementary space to $U\left(\mathrm{n}^{-}\right)_{-\mu+k \alpha} \bar{\theta}_{\alpha, k}$ in $U\left(\mathrm{n}^{-}\right)_{-\mu}$ constructed as in Shapovalov [23, p. 310]. Then as in [23] we have

$$
V_{-\mu} \oplus U\left(\mathfrak{n}^{-}\right)_{-\mu+k \alpha} \theta_{\alpha, k}(\xi)=U\left(\mathfrak{n}^{-}\right)_{-\mu} \text { for all } \mu \in Q^{+}, \xi \in \mathfrak{h}^{*} .
$$

Set $V=\bigoplus_{\mu \in Q^{+}} V_{-\mu}$.

Let for $\xi \in \mathfrak{h}_{\alpha, k}^{*}, V \stackrel{\psi_{\xi}}{\rightarrow} N(\xi)$ be the map given by $\psi_{\xi}(X)=X v_{\xi}+T(\xi) M(\xi-k \alpha)$. Then $\psi_{\xi}$ defines a linear isomorphism of $V$ with $N(\xi)$ for all $\xi \in \mathfrak{h}_{\alpha, k}^{*}$. Furthermore, $\psi_{\xi}$ is an $\mathfrak{h}$-module isomorphism of $\mathbf{C}(\xi) \otimes V$ onto $N(\xi)$.

Let for $X \in U(g), \pi_{\xi}(X) v=\psi_{\xi}^{-1}\left(X \psi_{\xi}(v)\right)$. Then $\left(\pi_{\xi}, V\right)$ is a family of representations of $\mathrm{g}$. We also note that if $X \in U(\mathrm{~g}), v \in V$ then $\xi \mapsto \pi_{\xi}(X) v$ defines a polynomial map of $\mathfrak{h}_{\alpha, k}^{*}$ into $\bigoplus_{\mu \in S} V_{-\mu}$ for some finite set $S \subset Q^{+}$depending only on $X$ and $v$.

We also note that for $\xi \in \mathfrak{h}_{\alpha, k}^{*},(,)_{\xi}$ induces a contravariant form on $M(\xi) / T(\xi)(M(\xi-k \alpha))$ since $T(\xi)(M(\xi-k \alpha))$ is contained in the radical of $(,)_{\xi}$. Thus we can pull back by $\psi_{\xi}$ the $(,)_{\xi}$ to define a family of forms $\langle,\rangle_{\xi}$ on $V$ such that:

(I) $\langle,\rangle_{\xi}$ is symmetric.

(II) $\langle,\rangle_{\xi}$ is nondegenerate if $\xi \in\left(\mathfrak{h}_{\alpha, k}^{*}\right)^{\prime}$. (In this case $N(\xi)=L(\xi)$.)

(III) If $v, w \in \dot{V}, \xi \mapsto\langle v, w\rangle_{\xi}$ is a polynomial map on $\mathfrak{h}_{\alpha, k}^{*}$.

(IV) $\left\langle\pi_{\xi}(X) v, w\right\rangle_{\xi}=\left\langle v, \pi_{\xi}(\sigma(X)) w\right\rangle_{\xi}$. (Here $\sigma$ is the antiautomorphism of $U(g)$ that fixes $h_{i}$ and sends $e_{i}$ to $f_{i}, i=1, \ldots, n$.) 
Let now $V[t]=\mathbf{C}[t] \otimes V$. We look upon $V[t]$ as the direct sum of the $\mathbf{C}[t] \otimes V_{-\mu}$ and upon $\mathbf{C}[t] \otimes V_{-\mu}$ as the polynomial maps of $\mathbf{C}$ into $V_{-\mu}$. Fix $\lambda \in \mathfrak{h}_{\alpha, k}^{*}$. We set

$$
V_{\lambda}^{i}[t]=\left\{f \in V[t] \mid\langle f(t), w\rangle_{\lambda+t \omega}=t^{i} h(t), h \in C[t]\right\} .
$$

By (I)-(IV) it is clear that if we set $V_{\lambda}^{i}=\left\{f(0) \mid f \in V_{\lambda}^{i}[t]\right\}$ then $V_{\lambda}^{i}$ is $\pi_{\lambda}$-invariant and $V \supset V_{\lambda}^{1} \supset V_{\lambda}^{2} \supset \cdots$. Furthermore, $V_{\lambda}^{1}$ is the radical of $\langle,\rangle_{\lambda}$. Thus $V / V_{\lambda}^{1}$ is isomorphic with $L(\lambda)$ under $\pi_{\lambda}$. Using $\psi_{\lambda}$ we have the desired filtration of $N(\lambda)$ : $N(\lambda)_{i}=\psi_{\lambda}\left(V_{\lambda}^{i}\right)$.

Note. It is easy to see that $N(\lambda)_{i}$ is independent of the choice of $V$. It is not known if it is independent of $\omega$.

TheOREM 3.10 (COMPARE JANTZen [10]). Let $\alpha \in \Delta_{R}^{+}$and $k \in \mathbf{Z}, k \geqslant 1$. Let $\lambda \in \mathfrak{h}_{\alpha, k}^{*}$ and let $N(\lambda)_{i}$ be as above. Then

$$
\begin{aligned}
\sum_{i \geqslant 1} \operatorname{ch~} N(\lambda)_{i} & =\sum_{\beta \in \Delta_{+}} \sum_{j>0, L_{j \beta}(\lambda)=0} \operatorname{ch} M(\lambda-j \beta) \\
& -\sum_{\gamma \in \Delta_{+}} \sum_{m>0, L_{m \gamma}(\lambda-k \alpha)=0} \operatorname{ch} M(\lambda-k \alpha-m \gamma)-a(\lambda) \operatorname{ch} M(\lambda-k \alpha)
\end{aligned}
$$

where $a(\lambda) \in \mathbf{Z}$ and $a(\lambda)=1$ if $\lambda \in V_{\alpha, k}$.

Proof. Let $\mu \in Q^{+}$. Fix $\left\{v_{1}, \ldots, v_{d_{\mu}}\right\}$ a basis of $V_{-\mu}$. Set for $\xi \in \mathfrak{h}_{\alpha, k}^{*}$

$$
D_{\xi-\mu}^{\prime}(\xi)=\operatorname{det}\left(\left\langle v_{i}, v_{j}\right\rangle_{\xi}\right)=\operatorname{det}\left(\left(v_{i}, v_{j}\right)_{\xi}\right) .
$$

Then Jantzen's argument in [10] implies

$$
\sum_{i \geqslant 1} \operatorname{ch} N(\lambda)_{i}=\sum_{\mu \in Q^{+}} e^{\lambda-\mu} \operatorname{ord}_{0}\left(D_{\lambda+t \omega-\mu}^{\prime}(\lambda+t \omega)\right) .
$$

Here ord ${ }_{0} f(t)$ is the largest power of $t$ dividing $f(t)$ for $f \in \mathbf{C}[t]$. We note that $\lambda+t \omega \in\left(\mathfrak{h}_{\alpha, k}^{*}\right)^{\prime}$ for $0<|t|<\varepsilon$ (some $\varepsilon>0$ ), so $D_{\lambda+t \omega-\mu}^{\prime}(\lambda+t \omega) \neq 0$ for $0<|t|<$ $\varepsilon, \mu \in Q^{+}$. We now compute the ord ${ }_{0}$.

Fix $\left\{u_{1}, \ldots, u_{s_{\mu}}\right\}$ a basis of $U\left(\mathfrak{n}^{-}\right)_{-\mu+k \alpha}$. Set $w_{i}(\xi)=v_{i}, i \leqslant d_{\mu}$, where $\left\{v_{1}, \ldots, v_{d_{\mu}}\right\}$ is a basis of $V_{-\mu}$, and set

$$
w_{i+d_{\mu}}(\xi)=u_{i} S_{-k \alpha}(\xi), \quad 1 \leqslant i \leqslant s_{\mu}=\mathcal{P}(\mu-k \alpha) .
$$

Here $\mathcal{P}: \mathfrak{h}^{*} \rightarrow \mathbf{Z}_{+}$is the generalized Kostant partition function defined as in [21]. Set $x_{i}=v_{i}, i \leqslant d_{\mu}$, and

$$
x_{i+d_{\mu}}=u_{i} \bar{\theta}_{\alpha, k}, \quad 1 \leqslant i \leqslant s_{\mu} .
$$

Then $w_{1}(\xi) \wedge \cdots \wedge w_{d_{\mu}+s_{\mu}}(\xi)=q_{k \alpha}(\xi)^{s_{\mu}} x_{1} \wedge \cdots \wedge x_{d_{\mu}+s_{\mu}}$

Thus if we set for $\xi \in \mathfrak{h}^{*}, D_{\xi-\mu}(\xi)=\operatorname{det}\left(\left(x_{i}, x_{j}\right)_{\xi}\right)$ then we have

$$
\operatorname{det}\left(w_{i}(\xi), w_{j}(\xi)\right)_{\xi}=q_{k \alpha}(\xi)^{2 s_{\mu}} D_{\xi-\mu}(\xi) .
$$


Lemma 3.8 implies that

$$
\begin{gathered}
{\left[\left(w_{i}(\xi), w_{j}(\xi)\right)_{\xi}\right] \quad \text { has block form: }} \\
{\left[\begin{array}{c|c}
\left(v_{i}, v_{j}\right)_{\xi} & r_{k \alpha}(\xi) Q(\xi) \\
\hline r_{k \alpha}(\xi) Q(\xi)^{t} & \left(u_{i} S_{-k \alpha}(\xi), u_{j} S_{-k \alpha}(\xi)\right)
\end{array}\right]\left(Q(\xi)^{t}=\text { transpose of } Q(\xi)\right) .}
\end{gathered}
$$

Hence if $\xi \in \mathfrak{h}^{*}$ then

$$
\begin{aligned}
q_{k \alpha}(\xi)^{2 s_{\mu}} D_{\xi-\mu}(\xi)= & \operatorname{det}\left(\left(v_{i}, v_{j}\right)_{\xi}\right) \operatorname{det}\left(\left(u_{i} S_{-k \alpha}(\xi), u_{j} S_{-k \alpha}(\xi)\right)_{\xi}\right) \\
& +r_{k \alpha}(\xi)^{s_{\mu}+1} h(\xi)
\end{aligned}
$$

where $h$ is a polynomial in $\xi$.

We note that $S_{-k \alpha}(\xi)-q_{k \alpha}(\xi) \theta_{\alpha, k}(\xi) \equiv 0$ on $\mathfrak{h}_{\alpha, k}^{*}$. Hence

$$
X S_{-k \alpha}(\xi) v_{\xi}=L_{k \alpha}(\xi) \delta(\xi) v_{\xi}, \quad X \in \mathfrak{n},
$$

where $\delta$ is a polynomial with values in $U\left(n^{-}\right)$. We therefore see that

$$
\begin{aligned}
\left(u_{i} S_{-k \alpha}(\xi), u_{j} S_{-k \alpha}(\xi)\right)_{\xi}= & \left(\sigma\left(u_{j}\right) u_{i} S_{-k \alpha}(\xi), S_{-k \alpha}(\xi)\right)_{\xi} \\
= & (\xi-k \alpha) p\left(\sigma\left(u_{j}\right) u_{i}\right)\left(S_{-k \alpha}(\xi), S_{-k \alpha}(\xi)\right)_{\xi} \\
& +L_{k \alpha}(\xi) r_{k \alpha}(\xi) \phi_{i j}(\xi)
\end{aligned}
$$

where $\phi_{i j}$ is a polynomial in $\xi$. Here $p: U(\mathfrak{g}) \rightarrow U(\mathfrak{h})$ is the Harish-Chandra projection corresponding to $U(\mathfrak{g})=U(\mathfrak{h}) \oplus\left(\mathfrak{n}^{-} U(\mathfrak{g})+U(\mathfrak{g}) \mathfrak{n}\right)$. Hence

$$
\begin{aligned}
\left(u_{i} S_{-k \alpha}(\xi),\right. & \left.u_{j} S_{-k \alpha}(\xi)\right)_{\xi} \\
& =\left(u_{i}, u_{j}\right)_{\xi-k \alpha} r_{k \alpha}(\xi) q_{k \alpha}(\xi)+L_{k \alpha}(\xi) r_{k \alpha}(\xi) \phi_{i j}(\xi)
\end{aligned}
$$

But this implies that up to a nonzero scalar multiple:

$$
\begin{aligned}
\operatorname{det}\left(\left(u_{i} S_{-k \alpha}(\xi)\right.\right. & \left.\left., u_{j} S_{-k \alpha}(\xi)\right)_{\xi}\right) \\
& =D_{\xi-k \alpha-\mu}(\xi-k \alpha) r_{k \alpha}(\xi)^{s_{\mu}} q_{k \alpha}(\xi)^{s_{\mu}}+L_{k \alpha}(\xi) r_{k \alpha}(\xi)^{s_{\mu}} h(\xi)
\end{aligned}
$$

with $h(\xi)$ a polynomial in $\xi$.

Dividing both sides of (3.9) by $\left(q_{k \alpha}(\xi) r_{k \alpha}(\xi)\right)^{s_{\mu}}$ and specializing to $\nu \in \mathfrak{h}_{\alpha, k}^{*}$ yields:

If $\nu \in \mathfrak{h}_{\alpha, k}^{*}$ then

$$
\left.q_{k \alpha}(\nu)^{s_{\mu}} \frac{D_{\xi-\mu}(\xi)}{r_{k \alpha}(\xi)^{s_{\mu}}}\right|_{\xi=\nu}=D_{\nu-\mu}^{\prime}(\nu) D_{\nu-k \alpha-\mu}(\nu-k \alpha)
$$

up to a nonzero scalar multiple. 
Kac and Kazhdan [14] have shown that

$$
D_{\xi-\mu}(\xi)=\prod_{\beta \in \Delta_{+}} \prod_{j=1}^{\infty} L_{j \beta}(\xi)^{\mathscr{P}(\mu-j \beta)}
$$

up to a nonzero scalar multiple.

Thus (3.13) combined with (3.14) yield using $s_{\mu}=\mathscr{P}(\mu-k \alpha)$ : If $\lambda \in \mathfrak{h}_{\alpha, k}^{*}$ then

$$
\begin{aligned}
\operatorname{Ord}_{0} D_{\lambda+t \omega-\mu}^{\prime}(\lambda+t \omega) & =\sum_{\beta \in \Delta^{+}} \sum_{j>0, L_{j \beta}(\lambda)=0} \mathscr{P}(\mu-j \beta) \\
& -\sum_{\gamma \in \Delta^{+}} \sum_{m>0, L_{m \gamma}(\lambda-k \alpha)=0} \mathscr{P}(\mu-k \alpha-m \gamma) \\
& +\mathscr{P}(\mu-k \alpha) \operatorname{ord}_{0} q_{k \alpha}(\lambda+t \omega)-\mathscr{P}(\mu-k \alpha) \operatorname{ord}_{0} r_{k \alpha}(\lambda+t \omega) .
\end{aligned}
$$

This says that if $k(\lambda)=\operatorname{ord}_{0}\left(r_{k \alpha}(\lambda+t \omega)\right)-1=\mid\left\{\gamma \mid \gamma=j \beta, j \in \mathbf{N}, \beta \in \Delta_{+}\right.$, $L_{\gamma}(\lambda)=0$ and $\left.j \beta<k \alpha, j \beta \neq \kappa \alpha\right\} \mid$ then: If $\lambda \in \mathfrak{h}_{\alpha, k}^{*}$

$$
\begin{aligned}
\sum_{i \geqslant 1} \operatorname{ch} N(\lambda)_{i}= & \sum_{\beta \in \Delta_{+}} \sum_{j>0, L_{j \beta}(\lambda)=0} \operatorname{ch} M(\lambda-j \beta) \\
& -\sum_{\beta \in \Delta_{+}} \sum_{j>0, L_{j \beta}(\lambda-k \alpha)=0} \operatorname{ch} M(\lambda-k \alpha-j \beta) \\
& +\left(\operatorname{ord}_{0}\left(q_{k \alpha}(\lambda+t \omega)\right)-k(\lambda)-1\right) \operatorname{ch} M(\lambda-k \alpha) .
\end{aligned}
$$

If $\lambda \in V_{\alpha, k}$ then $q_{k \alpha}(\lambda) \neq 0, k(\lambda)=0$. Thus (3.13) implies the theorem. Q.E.D.

Note. It is not hard to show that $a(\lambda) \in \mathbf{N}$.

4. Resolutions of irreducible modules over rank 2 Kac-Moody Lie algebras: An integral case. In this section we assume that $g$ is a symmetrizable GCM Lie algebra of rank 2, i.e. $\Delta_{0}=\left\{\alpha_{1}, \alpha_{2}\right\}$.

Let $W_{0}=\left\{\left(\sigma_{2} \sigma_{1}\right)^{j} \mid j \in \mathbf{Z}\right\} \subset W$. For each $k \in \mathbf{Z}_{+}$, set $w_{2 k}=\left(\sigma_{2} \sigma_{1}\right)^{k}, w_{2 k}^{\prime}=$ $\left(\sigma_{2} \sigma_{1}\right)^{-k}=\left(\sigma_{1} \sigma_{2}\right)^{k}, w_{2 k+1}=\sigma_{1}\left(\sigma_{2} \sigma_{1}\right)^{k}$ and $w_{2 k+1}^{\prime}=\sigma_{1}\left(\sigma_{2} \sigma_{1}\right)^{-(k+1)}=\sigma_{2}\left(\sigma_{1} \sigma_{2}\right)^{k}$.

The following is well known [2, Chapter IV, $\S 1$, Number 2, Proposition 2 and Remarque].

Proposition 4.1. (i) $W_{0}$ is a normal subgroup of $W$ of index 2 and $W$ is the semidirect product of the subgroup $\left\{1, \sigma_{1}\right\}$ and $W_{0}$.

(ii) Let $m$ be the order of $W_{0}$. If $m<\infty$ then $w_{0}=w_{0}^{\prime}, w_{r} \neq w_{r}^{\prime}, 1 \leqslant r \leqslant m-1$ and $w_{m}=w_{m}^{\prime}, W=\left\{w_{r}, w_{r}^{\prime} \mid 0 \leqslant r \leqslant m\right\}$ and $W^{(k)}=\left\{w_{k}, w_{k}^{\prime}\right\}, 0 \leqslant k \leqslant m$. Furthermore, every element of $W$ distinct from $w_{m}$ has a unique reduced decomposition. If $m=\infty$, $w_{0}=w_{0}^{\prime}$, the elements $w_{r}, w_{r}^{\prime}, r>0$, are all distinct, $W=\left\{w_{r}, w_{r}^{\prime}, r \geqslant 0\right\}$ and $W^{(k)}=$ $\left\{w_{k}, w_{k}^{\prime}\right\}, 0 \leqslant k$. Furthermore, every element of $W$ has a unique reduced decomposition. 
Let $m$ be the order of $W_{0}$. It follows from Proposition 4.1 and [21, Lemma 8.4], that the Bruhat ordering diagram of $W$ has one of the following forms (compare [10, 3.6] for the case $m<\infty)$ :
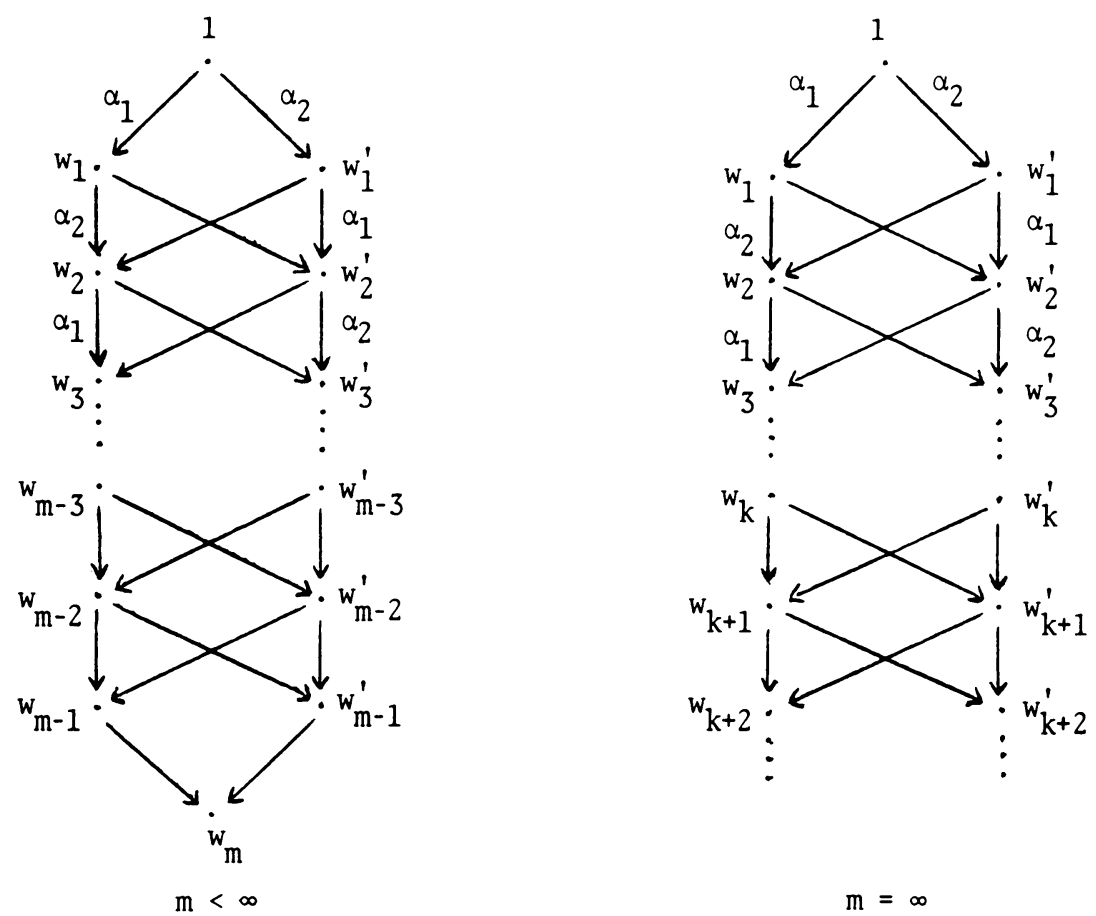

Proposition 4.2. Let $w, x \in W$. Then $x=\sigma_{\beta} w$ for some $\beta \in \Delta_{R}^{+}$if and only if $l(x)=l(w)+p, p \in \mathbf{Z}, p$ odd .

Proof. Let $\gamma \in \Delta_{R}^{+}$. Let $w_{1} \in W$ and $i \in\{1,2\}$ be such that $\gamma=w_{1} \alpha_{i}$. Then $\sigma_{\gamma}=w_{1} \sigma_{i} w_{i}^{-1}$. If $l\left(w_{1}\right)=r$ then $l\left(\sigma_{\gamma}\right)=2 r+1$ or $l\left(\sigma_{\gamma}\right)=2 r-1$, by Proposition 4.1. Hence $l\left(\sigma_{\gamma}\right)$ is odd. If $x=\sigma_{\beta} w, \beta \in \Delta_{R}^{+}$, then the same argument shows that $l(x)=l(w)+l\left(\sigma_{\beta}\right)$ or $l(x)=l(w)+l\left(\sigma_{\beta}\right)-2$. Hence $l(x)=l(w)+p, p$ odd. Conversely, suppose $l(x)=l(w)+p, p$ odd. Assume $p>0$. Let $w=\sigma_{k_{1}} \cdots \sigma_{k_{t}}$ and $x=\sigma_{i_{1}} \cdots \sigma_{i_{r}}$ be reduced expressions. Let $q$ be the smallest index such that $i_{q} \neq k_{q}$. Then $w^{\prime}=x^{-1} w=\sigma_{i_{r}} \cdots \sigma_{i_{q}} \sigma_{k_{q}} \cdots \sigma_{k_{t}}$ is reduced and $l\left(w^{\prime}\right)=2[l(w)-(q-1)]+$ $p$, hence odd. Let $w^{\prime}=\sigma_{j_{1}} \cdots \sigma_{j_{m}}$. If $m=1$, then $x=\sigma_{j_{1}} w$. If $m>1$, set $s=$ $(m-1) / 2$ and $w_{1}=\sigma_{j_{1}} \cdots \sigma_{j_{s}}$. Then $w^{\prime}=w_{1} \sigma_{j_{s+1}} w_{1}^{-1}=\sigma_{\beta}$, where $\beta=w_{1} \alpha_{j_{s+1}}$. If $l(x)=l(w)+p, p$ odd, $p<0$, then $l(w)=l(x)+a, a$ odd, $a>0$. By the above, $w=\sigma_{\beta} x$ where $\beta \in \Delta_{R}^{+}$. Hence $x=\sigma_{\beta} w, \beta \in \Delta_{R}^{+}$. Q.E.D.

Proposition 4.3. Let $X$ be a standard module with highest weight $\mu \in P^{+}$. There is a resolution of $X$ :

$$
\cdots \rightarrow M\left(w_{j} \cdot \mu\right) \oplus M\left(w_{j}^{\prime} \cdot \mu\right) \stackrel{\alpha_{j}}{\rightarrow} \cdots \stackrel{\alpha_{2}}{\rightarrow} M\left(w_{1} \cdot \mu\right) \oplus M\left(w_{1}^{\prime} \cdot \mu\right) \stackrel{\alpha_{1}}{\rightarrow} M(\mu) \stackrel{\varepsilon}{\rightarrow} X \rightarrow 0
$$


where $\alpha_{j}(x, y)=\left(i_{w_{j}, w_{j-1}} x+i_{w_{j}^{\prime}, w_{j-1}} y,-i_{w_{j}, w_{j-1}^{\prime}} x-i_{w_{j}^{\prime}, w_{j-1}^{\prime}} y\right)$, for $x \in M\left(w_{j} \cdot \mu\right), y \in$ $M\left(w_{j}^{\prime} \cdot \mu\right), j>1, \alpha_{1}(x, y)=i_{w_{1}, 1} x+i_{w_{1}^{\prime}, 1} y, x \in M\left(w_{1} \cdot \mu\right), y \in M\left(w_{1}^{\prime} \cdot \mu\right)$.

Proof. The sequence (4.1) is clearly a complex, by Theorem 2.4 and the Bruhat ordering diagram above. Hence (4.1) is exact by Scholium 2.6. Q.E.D.

REMARK. If the order of $W_{0}$ is finite and equals $m$ then the resolution (4.1) ends and $\alpha_{m}: \quad M\left(w_{m} \cdot \mu\right) \rightarrow M\left(w_{m-1} \cdot \mu\right) \oplus M\left(w_{m-1}^{\prime} \cdot \mu\right)$ is defined by: $\alpha_{m}(x)=$ $\left(i_{w_{m}, w_{m-1}} x,-i_{w_{m}, w_{m-1}^{\prime}} x\right), x \in M\left(w_{m} \cdot \mu\right)$.

We now recall Jantzen's filtration and character formula which are valid for $(\mathfrak{g}, \mathfrak{h})$ of arbitrary rank. Let $\Delta_{+}(\chi)=\left\{\beta \in \Delta_{+} \mid 2(\chi+\rho, \beta)=i(\beta, \beta), i \in \mathbf{N}\right\}, \chi \in \mathfrak{h}^{*}$ and $\mathbf{N}_{\beta}(\chi)=\{i \in \mathbf{N} \mid 2(\chi+\rho, \beta)=i(\beta, \beta)\}$.

Proposition 4.4 [10, Satz 5.3 And 14, (4.2)]. Let $\chi \in \mathfrak{h}^{*}$. There is a filtration of submodules $M(\chi)=M(\chi)_{0} \supset M(\chi)_{1} \supset \cdots$ such that

(i) $M(\chi)_{1}$ is the largest proper submodule of $M(\chi)$;

(ii) for every $i \in \mathbf{N}$ there is on $M(\chi)_{i} / M(\chi)_{i+1}$ a nondegenerate contravariant form $(,)_{i}$; furthermore

(iii) $\Sigma_{i>0} \operatorname{ch} M(\chi)_{i}=\Sigma_{\alpha \in \Delta_{+}(\chi)} \Sigma_{i \in N_{\alpha}(\chi)} \operatorname{ch} M(\chi-i \alpha)$.

REMARK. The filtration $M(\chi) \supset M(\chi)_{1} \supset \cdots$ was defined by extending the base field $\mathbf{C}$ to the ring of polynomials in one variable over $\mathbf{C}$. Here, we will give a construction of this filtration directly from the structure of $M(\chi), \chi \in W \cdot P^{+}$, and therefore will answer various questions posed in [10, 5.17] about the $M(\chi)_{i}$, $\chi \in W \cdot P^{+}$, in the rank 2 situation.

Corollary 4.5. Let $\nu \in W \cdot P^{+}$. Then

$$
\sum_{i \geqslant 1} \operatorname{ch} M(\nu)_{i}=\sum_{\alpha \in \Delta_{+}(\nu)} \operatorname{ch} M\left(\sigma_{\alpha} \cdot \nu\right) .
$$

Proof. This follows from Proposition 4.4(iii) and Lemma 2.1. Q.E.D.

Definition 4.6. Let $\mu \in P^{+}$. We define $M(\mu)^{i}=M\left(w_{i} \cdot \mu\right)+M\left(w_{i}^{\prime} \cdot \mu\right), i \in \mathbf{N}$. If $\nu \in W^{(k)} \cdot \mu$ we define $M(\nu)^{i}=M\left(w_{k+i} \cdot \mu\right)+M\left(w_{k+i}^{\prime} \cdot \mu\right)=M(\mu)^{k+i}$.

Proposition 4.7. $M\left(w_{i} \cdot \mu\right)+M\left(w_{i}^{\prime} \cdot \mu\right)=M\left(w_{i-1} \cdot \mu\right) \cap M\left(w_{i-1}^{\prime} \cdot \mu\right)$, for $\mu \in P^{+}$, $i=1,2, \ldots$ If $\nu \in W \cdot P^{+}$then $M(\nu) \supset M(\nu)^{1} \supset M(\nu)^{2} \supset \cdots$ is a g-module filtration and

$$
\sum_{i \geqslant 1} \operatorname{ch} M(\nu)^{i}=\sum_{\alpha \in \Delta_{+}(\nu)} \operatorname{ch} M\left(\sigma_{\alpha} \cdot \nu\right)
$$

Proof. The first statement follows from the exactness of (4.1). The statement that $M(\nu) \supset M(\nu)^{1} \supset M(\nu)^{2} \supset \cdots$ is a g-module filtration follows from Theorem 2.4. Let $\nu \in W^{(k)} \cdot \mu$. From

$$
\begin{aligned}
\operatorname{ch}\left(M\left(w_{i} \cdot \mu\right)\right. & \left.+M\left(w_{i}^{\prime} \cdot \mu\right)\right) \\
& =\operatorname{ch} M\left(w_{i} \cdot \mu\right)+\operatorname{ch} M\left(w_{i}^{\prime} \cdot \mu\right)-\operatorname{ch} M\left(w_{i} \cdot \mu\right) \cap M\left(w_{i}^{\prime} \cdot \mu\right)
\end{aligned}
$$

for all $i \geqslant 1$ and from the first statement it follows that $\Sigma_{i \geqslant 1} \operatorname{ch} M(\nu)^{i}=$ $\Sigma_{i=k+l} \operatorname{ch} M\left(w_{i} \cdot \mu\right)+\operatorname{ch} M\left(w_{i}^{\prime} \cdot \mu\right), l \in \mathrm{N}, l$ odd. Let $\nu=w \cdot \mu, w \in W^{(k)}$. By 
Proposition 4.2, $w_{i}=\sigma_{\alpha} w$ for some $\alpha \in \Delta_{R}^{+}$if and only if $i=k+p, p \in \mathbf{Z}, p$ odd. Now, $2(\nu+\rho, \alpha)=i(\alpha, \alpha)$ with $i \in \mathbf{N}, \alpha \in \Delta^{+}$if and only if $\alpha \in \Delta_{R}^{+}$and $l\left(\sigma_{\alpha} w\right)>$ $l(w)$, by Lemma 2.1. Hence, $\sum_{i=k+l, l \in \mathrm{N}, l \text { odd }} \operatorname{ch} M\left(w_{i} \cdot \mu\right)+\operatorname{ch} M\left(w_{i}^{\prime} \cdot \mu\right)=$ $\Sigma_{\alpha \in \Delta_{+}(\nu)} \operatorname{ch} M\left(\sigma_{\alpha} \cdot \nu\right)$. Q.E.D.

Definition 4.8. Let $\nu \in W \cdot P^{+}$. We define $\tilde{L}(\nu)=M(\nu) / M(\nu)^{1}$.

Proposition 4.9. Let $\nu=w \cdot \mu, \mu \in P^{+}, w \in W^{(k)}$. There exists a resolution of $\tilde{L}(\nu)$ :

$$
\begin{gathered}
\cdots \rightarrow M\left(w_{i} \cdot \mu\right) \oplus M\left(w_{i}^{\prime} \cdot \mu\right) \stackrel{\alpha_{i}}{\rightarrow} \cdots \stackrel{\alpha_{k+2}}{\rightarrow} M\left(w_{k+1} \cdot \mu\right) \oplus M\left(w_{k+1}^{\prime} \cdot \mu\right) \\
\stackrel{\eta_{k+1}}{\rightarrow} M(\nu) \stackrel{\varepsilon_{k}}{\rightarrow} \tilde{L}(\nu) \rightarrow 0
\end{gathered}
$$

where the $\alpha_{i}, i \geqslant k+2$, are defined as in Proposition $4.3, \eta_{k+1}(x, y)=i_{w_{k+1}, w} x+$ $i_{w_{k+1}^{\prime}, w} y$ and $\varepsilon_{k}$ is the canonical surjection.

Proof. By Proposition 4.3 it suffices to check exactness at $M\left(w_{k+1} \cdot \mu\right) \oplus$ $M\left(w_{k+1}^{\prime} \cdot \mu\right)$ and at $M(w \cdot \mu)$.

$$
\begin{aligned}
\operatorname{Ker} \varepsilon_{k} & =M(w \cdot \mu)^{1}=M(\mu)^{k+1} \\
& =M\left(w_{k+1} \cdot \mu\right)+M\left(w_{k+1}^{\prime} \cdot \mu\right)=\text { image of } \eta_{k+1} .
\end{aligned}
$$

$\operatorname{Ker} \eta_{k+1}=\left\{(u,-u) \mid u \in M\left(w_{k+1} \cdot \mu\right) \cap M\left(w_{k+1}^{\prime} \cdot \mu\right)\right\}=M\left(w_{k+2} \cdot \mu\right)+$ $M\left(w_{k+2}^{\prime} \cdot \mu\right)$, by Proposition 4.7. Hence Ker $\eta_{k+1}=$ Image of $\alpha_{k+2}$. Q.E.D.

Next, we will show that the $\tilde{L}(w \cdot \mu)$ are irreducible, $\mu \in P^{+}, w \in W$.

Definition 4.10. Let $\mu \in P^{+}, w \in W^{(k)}, w^{\prime} \in W^{(k+1)}$. Set $N\left(w, w^{\prime} ; \mu\right)=$ $M(w \cdot \mu) / M\left(w^{\prime} \cdot \mu\right), \quad N\left(w, w^{\prime} ; \mu\right)^{1}=\left(M\left(w_{k+1} \cdot \mu\right)+M\left(w_{k+1}^{\prime} \cdot \mu\right)\right) / M\left(w^{\prime} \cdot \mu\right)$ and $N\left(w, w^{\prime} ; \mu\right)^{i}=0, i>1$.

Lemma 4.11. Let $\mu \in P^{+}, w \in W$. Then $\tilde{L}(w \cdot \mu)$ is irreducible.

Proof. Let $w \in W^{(k)}, w^{\prime} \in W^{(k+1)}$ and $N\left(w, w^{\prime} ; \mu\right) \supset N\left(w, w^{\prime} ; \mu\right)^{1}$ be as above. $\operatorname{Then} \operatorname{ch}\left(N\left(w, w^{\prime} ; \mu\right) / N\left(w, w^{\prime} ; \mu\right)^{1}\right)=\operatorname{ch} \tilde{L}(w \cdot \mu)$. Now

$$
\sum_{i>0} \operatorname{ch} N\left(w, w^{\prime} ; \mu\right)^{i}=\operatorname{ch} N\left(w, w^{\prime} ; \mu\right)^{1}=\operatorname{ch} \tilde{L}\left(w_{k+1}^{\prime} \cdot \mu\right)
$$

(resp. $\left.\operatorname{ch} \tilde{L}\left(w_{k+1} \cdot \mu\right)\right)$ if $w^{\prime}=w_{k+1}$ (resp. if $\left.w^{\prime}=w_{k+1}^{\prime}\right)$. By Theorem 3.10 and Proposition 4.7,

$$
\begin{aligned}
\sum_{i>0} \operatorname{ch} N\left(w, w^{\prime} ; \mu\right)_{i}= & \sum_{\substack{i \in \mathbf{N} \\
i \text { odd }}}\left(\operatorname{ch} M\left(w_{k+i} \cdot \mu\right)+\operatorname{ch} M\left(w_{k+i}^{\prime} \cdot \mu\right)\right) \\
& -\sum_{\substack{i \in \mathbf{N} \\
i \text { odd }}}\left(\operatorname{ch} M\left(w_{k+1+i} \cdot \mu\right)+\operatorname{ch} M\left(w_{k+1+i}^{\prime} \cdot \mu\right)\right)-\operatorname{ch} M\left(w^{\prime} \cdot \mu\right) \\
= & \operatorname{ch} \tilde{L}\left(w_{k+1}^{\prime} \cdot \mu\right) \quad\left(\operatorname{resp} . \operatorname{ch} \tilde{L}\left(w_{k+1} \cdot \mu\right)\right)
\end{aligned}
$$

if $w^{\prime}=w_{k+1}$ (resp. if $\left.w^{\prime}=w_{k+1}^{\prime}\right)$. Now, $N\left(w, w^{\prime} ; \mu\right)_{i} \supset N\left(w, w^{\prime} ; \mu\right)^{i}$ for all $i>0$. Set $q_{i}=\operatorname{ch}\left(N\left(w, w^{\prime} ; \mu\right)_{i} / N\left(w, w^{\prime} ; \mu\right)^{i}\right), i>0$. Assume that $q_{i} \neq 0$ for some $i>0$. Then $\Sigma_{i>0} \operatorname{ch} N\left(w, w^{\prime} ; \mu\right)^{i}+\Sigma_{i>0} q_{i}=\sum_{i>0} \operatorname{ch} N\left(w, w^{\prime} ; \mu\right)_{i}$. The above implies that 
$\sum_{i>0} q_{i}=0$, a contradiction. Hence $q_{i}=0$ for all $i>0$. Therefore $N\left(w, w^{\prime} ; \mu\right)^{i}=$ $N\left(w, w^{\prime} ; \mu\right)_{i}$ for all $i>0$. In particular, $N\left(w, w^{\prime} ; \mu\right)^{1}=N\left(w, w^{\prime} ; \mu\right)_{1}$. Hence $N\left(w, w^{\prime} ; \mu\right) / N\left(w, w^{\prime} \cdot \mu\right)^{1}$ is irreducible and thus $\tilde{L}(w \cdot \mu)$ is irreducible. Q.E.D.

REMARK. By the Bruhat ordering diagram, given $w \in W^{(k)}$ there is $w^{\prime} \in W^{(k+1)}$ such that $w^{\prime} \stackrel{\alpha}{\leftarrow} w$, with $\alpha \in \Delta_{0}$. In this case the proof of Theorem 3.10 for $N\left(w, w^{\prime} ; \mu\right)$ is considerably simpler. Therefore the proof of Lemma 4.11 can be simplified by making such a choice of $w^{\prime}$ and using this much simpler version of Theorem 3.10. However we will need Theorem 3.10 in its full generality in $\$ 5$.

THeOREM 4.12. Let $\mu \in P^{+}, w \in W^{(k)}$. There exists a resolution of $L(w \cdot \mu)$ :

$$
\begin{aligned}
\cdots \rightarrow M\left(w_{i} \cdot \mu\right) \oplus M\left(w_{i}^{\prime} \cdot \mu\right) \rightarrow \cdots & \stackrel{\alpha_{k+2}}{\rightarrow} M\left(w_{k+1} \cdot \mu\right) \oplus M\left(w_{k+1}^{\prime} \cdot \mu\right) \\
& \stackrel{\eta_{k+1}}{\rightarrow} M(w \cdot \mu) \stackrel{\varepsilon_{k}}{\rightarrow} L(w \cdot \mu) \rightarrow 0
\end{aligned}
$$

where the $\alpha_{i}, i \geqslant k+2, \eta_{k+1}$ and $\varepsilon_{k}$ are as in Proposition 4.9.

Proof. This follows from Proposition 4.9 and Lemma 4.11. Q.E.D.

The following corollary is now immediate.

Corollary 4.13. Let $\mu \in P^{+}$. For each $w \in W^{(k)}$ we have

$$
\operatorname{ch} L(w \cdot \mu)=\operatorname{ch} M(w \cdot \mu)+(-1)^{k} \sum_{i>k}(-1)^{i}\left(\operatorname{ch} M\left(w_{i} \cdot \mu\right)+\operatorname{ch} M\left(w_{i}^{\prime} \cdot \mu\right)\right),
$$

$$
\operatorname{ch} M(w \cdot \mu)=\operatorname{ch} L(w \cdot \mu)+\sum_{i>k}\left(\operatorname{ch} L\left(w_{i} \cdot \mu\right)+\operatorname{ch} L\left(w_{i}^{\prime} \cdot \mu\right)\right)
$$

COROllaRY 4.14. Let $\mu \in P^{+}, w, w^{\prime} \in W$. Then:

(i) $\left(M(w \cdot \mu): L\left(w^{\prime} \cdot \mu\right)\right)=1\left(\right.$ resp. 0) if $w^{\prime} \leqslant w($ resp. otherwise $)$;

(ii) $\left(I\left(w^{\prime} \cdot \mu\right): M(w \cdot \mu)\right)=1$ (resp. 0) if $w^{\prime} \leqslant w$ (resp. otherwise).

Proof. (i) follows from Theorem 4.12. (ii) follows from (i) and Theorem 1.2. Q.E.D.

LEMMA 4.15. Let $\mu \in P^{+}, w \in W^{(k)}$. If $M(w \cdot \mu)_{j}=M(w \cdot \mu)^{j}$ for $1 \leqslant j \leqslant i$ then $M(w \cdot \mu)_{i} / M(w \cdot \mu)_{i+1}=L\left(w_{k+i} \cdot \mu\right) \oplus L\left(w_{k+i}^{\prime} \cdot \mu\right)$.

Proof. Let $W^{(k+i)}=\{s, t\}$. By (4.2) and Proposition 4.7 we obtain $\Sigma_{j>i} \operatorname{ch} M(w \cdot \mu)_{j}=\Sigma_{l \in \mathrm{N}, ~ l o d d}\left(\operatorname{ch} M\left(w_{k+i+l} \cdot \mu\right)+\operatorname{ch} M\left(w_{k+i+l}^{\prime} \cdot \mu\right)\right)$. Let $v_{k+i}$ (resp. $\left.u_{k+i}\right)$ be a nonzero vector of weight $s \cdot \mu(\operatorname{resp} . t \cdot \mu)$ such that $M(w \cdot \mu)_{i}=U(g) v_{k+i}$ $+U(g) u_{k+i}$. From the formula above it follows that $v_{k+i}, u_{k+i} \notin M(w \cdot \mu)_{i+1}$. Let $\pi: M(w \cdot \mu)_{i} \rightarrow M(w \cdot \mu)_{i} / M(w \cdot \mu)_{i+1}$ be the canonical surjection. Then $M(w \cdot \mu)_{i} / M(w \cdot \mu)_{i+1}=U(\mathfrak{g}) \pi\left(v_{k+i}\right)+U(\mathfrak{g}) \pi\left(u_{k+i}\right)$. Set $M_{k+i}=U(\mathfrak{g}) \pi\left(v_{k+i}\right)$ and $M_{k+i}^{\prime}=U(\mathfrak{g}) \pi\left(u_{k+i}\right)$. Let us assume that $s \cdot \mu \& t \cdot \mu$. Then $\left(M(w \cdot \mu)_{i} / M(w \cdot \mu)_{i+1}\right)_{s \cdot \mu}=\left(M_{k+i}\right)_{s \cdot \mu}$. Hence, the restriction to $M_{k+i}$ of the nondegenerate contravariant form $(,)_{k}$ is nondegenerate. Let $M \subset M_{k+i}$ be a proper 
submodule. Then $M_{s \cdot \mu}=0$, hence $\left(M_{k+i}\right)_{s \cdot \mu} \subset M^{\perp}$, where $M^{\perp}=\left\{v \in M_{k+i} \mid\right.$ $(v, u)_{i}=0$ for all $\left.u \in M\right\}$. Therefore $M_{k+i}=M^{\perp}$ and so $M=(0)$. Hence, $M_{k+i}=$ $L(s \cdot \mu)$ and $M(w \cdot \mu)_{i} / M(w \cdot \mu)_{i+1}=L(s \cdot \mu) \oplus M_{k+i}^{\prime}$. Let $v \in M_{k+i}^{\prime}$ be such that $(v, u)_{i}=0$ for all $u \in M_{k+i}^{\prime}$. Now $\left(v, v_{k+i}\right)_{i}=0$ by weight considerations. Hence $(v, z)_{i}=0$ for all $z \in M(w \cdot \mu)_{i} / M(w \cdot \mu)_{i+1}$ and therefore $v=0$. So the restriction of $(,)_{i}$ to $M_{k+i}^{\prime}$ is nondegenerate. By the above argument, $M_{k+i}^{\prime}=L(t \cdot \mu)$, and so $M(w \cdot \mu)_{i} / M(w \cdot \mu)_{i+1}=L\left(w_{k+i} \cdot \mu\right) \oplus L\left(w_{k+i}^{\prime} \cdot \mu\right)$. Q.E.D.

THeOREM 4.16. Let $\mu \in P^{+}, w \in W^{(k)}$. Then:

(i) $M(w \cdot \mu)_{i}=M\left(w_{k+i} \cdot \mu\right)+M\left(w_{k+i}^{\prime} \cdot \mu\right)$;

(ii) $M(w \cdot \mu)_{i} / M(w \cdot \mu)_{i+1}=L\left(w_{k+i} \cdot \mu\right) \oplus L\left(w_{k+i}^{\prime} \cdot \mu\right)$.

Proof. By Lemma $4.11 M(w \cdot \mu)_{1}=M\left(w_{k+1} \cdot \mu\right)+M\left(w_{k+1}^{\prime} \cdot \mu\right)=M(w \cdot \mu)^{1}$. Suppose we have shown that $M(w \cdot \mu)^{j}=M(w \cdot \mu)_{j}$ for all $j, 1 \leqslant j<i$. Then $M(w \cdot \mu)_{j} / M(w \cdot \mu)_{j+1}=L\left(w_{k+j} \cdot \mu\right) \oplus L\left(w_{k+j}^{\prime} \cdot \mu\right)$ for $1 \leqslant j<i$, by Lemma 4.15. By Corollary 4.14(i) it follows that $M(w \cdot \mu)^{i} \subset M(w \cdot \mu)_{i}$. Our induction assumption and Proposition 4.7 imply that

$$
\sum_{j>i-2} \operatorname{ch} M(w \cdot \mu)_{j}=\sum_{\substack{l \in \mathbf{N} \\ l \text { odd }}}\left(\operatorname{ch} M\left(w_{k+i-2+l} \cdot \mu\right)+\operatorname{ch} M\left(w_{k+i-2+l}^{\prime} \cdot \mu\right)\right) .
$$

Hence

$$
\begin{aligned}
\operatorname{ch} L\left(w_{k+i-1} \cdot \mu\right)+\operatorname{ch} L\left(w_{k+i-1}^{\prime} \cdot \mu\right)+\operatorname{ch} M(w \cdot \mu)_{i}+\sum_{j>i-1} \operatorname{ch} M(w \cdot \mu)_{j} \\
=\operatorname{ch} L\left(w_{k+i-1} \cdot \mu\right)+\operatorname{ch} M\left(w_{k+i-1} \cdot \mu\right)_{1} \\
+\operatorname{ch} L\left(w_{k+i-1}^{\prime} \cdot \mu\right)+\operatorname{ch} M\left(w_{k+i-1}^{\prime} \cdot \mu\right)_{1} \\
+\sum_{\substack{l \in N \\
l \text { odd }}}\left(\operatorname{ch} M\left(w_{k+i+l} \cdot \mu\right)+\operatorname{ch} M\left(w_{k+i+l}^{\prime} \cdot \mu\right)\right) .
\end{aligned}
$$

Therefore,

$$
\begin{aligned}
\operatorname{ch} M(w \cdot \mu)_{i}+\sum_{j>i-1} \operatorname{ch} M(w \cdot \mu)_{j} \operatorname{ch} M\left(w_{k+i-1} \cdot \mu\right)_{1}+\operatorname{ch} M\left(w_{k+i-1}^{\prime} \cdot \mu\right)_{1} \\
\quad+\sum_{\substack{l \in \mathbf{N} \\
l \text { odd }}}\left(\operatorname{ch} M\left(w_{k+i+l} \cdot \mu\right)+\operatorname{ch} M\left(w_{k+i+l}^{\prime} \cdot \mu\right)\right) \\
=\operatorname{ch} M\left(w_{k+i-1} \cdot \mu\right)_{1}+\operatorname{ch}\left(M\left(w_{k+i} \cdot \mu\right)+M\left(w_{k+i}^{\prime} \cdot \mu\right)\right) \\
\quad+\sum_{l \in \mathbf{N}, l \text { odd }}\left(\operatorname{ch~} M\left(w_{k+i+l} \cdot \mu\right)+\operatorname{ch} M\left(w_{k+i+l}^{\prime} \cdot \mu\right)\right) \\
=\operatorname{ch} M\left(w_{k+i-1} \cdot \mu\right)_{1}+\sum_{l \in \mathbf{N}, l \text { odd }}\left(\operatorname{ch} M\left(w_{k+i-1+l} \cdot \mu\right)+\operatorname{ch} M\left(w_{k+i-1+l}^{\prime} \cdot \mu\right)\right),
\end{aligned}
$$

by Proposition 4.7. Thus

$\operatorname{ch} M(w \cdot \mu)_{i}=\operatorname{ch} M\left(w_{k+i-1} \cdot \mu\right)_{1}=\operatorname{ch}\left(M\left(w_{k+i} \cdot \mu\right)+M\left(w_{k+i}^{\prime} \cdot \mu\right)\right)=\operatorname{ch} M(w \cdot \mu)^{i}$.

Hence (i); (ii) follows from (i) and Lemma 4.15. Q.E.D. 
REMARKS. (1) Theorem 4.16(i) implies that Jantzen's filtration is hereditary: Let $\mu \in P^{+}, w, w^{\prime} \in W$. If $l(w)-l\left(w^{\prime}\right)=r \in \mathbf{N}$ then $M(w \cdot \mu)_{i}=M\left(w^{\prime} \cdot \mu\right)_{r+i}$, all $i \in \mathbf{N}$. Our arguments also show that (4.5), (4.6), the exactness of (4.4), the statements (i) and (ii) of Corollary 4.14, the statements (i) and (ii) of Theorem 4.16 and the above statement on the heredity of Jantzen's filtration are all equivalent statements.

(2) The results of this section generalize the results of Jantzen $[10,3.17,5.5]$ in the case where $\lambda$ is regular integral; we do not use Bernstein varieties [10, §3], but instead we make full use of our resolution and the a priori proof of Theorem 3.10. In $\$ 5$ we include the singular integral as well as the nonintegral case.

(3) Corollary 4.13 proves the Kazhdan-Lusztig conjecture [15] in our case.

(4) In $\$ 5$ we extend the results of this section to include the case where $\mu+\rho \in P^{+}$.

5. Resolutions of irreducible modules over rank 2 Kac-Moody Lie algebras: A complementary case. We keep the notation of $\S 2$. Let $\chi \in \mathfrak{h}^{*}$. Set $\Delta(\chi)=\{\alpha \in \Delta \mid$ $2(\chi+\rho, \alpha)=m(\alpha, \alpha)$ for some $m \in \mathbf{Z}\}$. We denote by $\Delta_{\chi}$ the smallest root subsystem of $\Delta$ containing $\Delta(\chi)$ and such that the real roots $\left(\Delta_{\chi}\right)_{R}$ of $\Delta_{\chi}$ are the roots of $\Delta_{\chi}$ that are real in $\Delta$. That is, $\Delta_{\chi}$ is the root system of some GCM Lie algebra such that $\Delta_{\chi} \supset \Delta(\chi),\left(\Delta_{\chi}\right)_{R}=\Delta_{\chi} \cap \Delta_{R}$ and if $\Delta^{\prime}$ is a subset of $\Delta$ with these properties then $\Delta^{\prime} \supset \Delta_{x}$. Let $\Delta_{x}^{+}=\Delta_{+} \cap \Delta_{x}, \Delta_{x}^{0}=\left\{\alpha \in \Delta_{\chi}^{+} \mid \alpha \neq \gamma_{1}+\cdots+\gamma_{k}\right.$ for all $\left.\gamma_{i} \in \Delta_{x}^{+}, i=1, \ldots, k, k \geqslant 2\right\}$, and set $\Delta_{x}^{0}=\left\{\beta_{1}, \ldots, \beta_{l}\right\}$. Let $W_{x}$ be the subgroup of $W$ generated by the $\sigma_{\beta_{i}}, i=1, \ldots, l$.

REMARK. In the semisimple case $\Delta_{\chi}=\Delta(\chi)[10,1.3]$.

Let $l_{\chi}$ denote the length function in $W_{\chi}$ and let $\leqslant_{\chi}$ be the Bruhat order in $W_{\chi}$.

We make the following assumption on $\chi$ :

(A) There exists $s_{0} \in W_{\chi}$ such that $2\left(s_{0}(\chi+\rho), \beta_{i}\right)=m_{i}\left(\beta_{i}, \beta_{i}\right)$ with $-m_{i} \in \mathbf{Z}_{+}$, $i=1, \ldots, l$ and

$$
\left(s_{0}(\chi+\rho), \beta\right) \neq 0 \quad \text { if }(\beta, \beta)=0, \beta \in \Delta_{\chi} .
$$

Set $\chi_{0}=s_{0} \cdot \chi$. Using the same argument as in the proof of Lemma 2.1 [21, Lemma 8.2], we obtain

LEMma 5.1. Let $w \in W_{\chi}$ and $\beta \in \Delta_{\chi}^{+}$be such that $(\beta, \beta) \geqslant 0$. Then the following statements are equivalent:

(i) $2\left(w\left(\chi_{0}+\rho\right), \beta\right)=m(\beta, \beta)$ with $m \in \mathbf{N}$,

(ii) $\beta \in \cup_{i=1}^{l} W_{\chi} \beta_{i}$ and $l_{\chi}(w)>l_{\chi}\left(\sigma_{\beta} w\right)$.

Let $X=\left\{\beta_{i} \mid\left(\chi_{0}+\rho, \beta_{i}\right)=0\right\} \subset \Delta_{x}^{0}$. We denote by $W_{x, X}$ the subgroup of $W_{\chi}$ generated by $\sigma_{\beta}$ with $\beta \in X$. Let $W_{\chi, X}^{1}$ denote the set of elements $w$ in $W_{\chi}$ of minimal length $l_{\chi}(w)$ among the representatives of the coset $w W_{\chi, X}$. Every element of $W_{\chi}$ can be uniquely expressed in the form $w^{1} w$ where $w \in W_{\chi, X}, w^{1} \in W_{\chi, X}^{1}$ and $l_{\chi}\left(w^{1} w\right)=$ $l_{\chi}\left(w^{1}\right)+l_{\chi}(w)$. 
Using Lemma 5.1 and the argument of [21, Lemma 8.7] we obtain

Lemma 5.2. Let $\chi$ be such that $(\beta, \beta) \geqslant 0$ for all $\beta \in \Delta_{\chi}$ and let $w, w^{\prime} \in W_{\chi, X}^{1}$. Then:

(i) $\left(M\left(w \cdot \chi_{0}\right): L(\psi)\right) \neq 0$ if and only if $\psi=w^{\prime} \cdot \chi_{0}$ for some $w^{\prime} \in W_{\chi}$ with $w \leqslant_{\chi} w^{\prime}$

(ii) If $\left(M\left(w \cdot \chi_{0}\right): L(\psi)\right) \neq 0, w \neq w^{\prime}, w^{\prime}$ as in (i) then there are $w_{0}, w_{1}, \ldots, w_{r} \in$ $W_{\chi, X}^{1}$ such that $l_{\chi}\left(w_{i+1}\right)=l_{\chi}\left(w_{i}\right)+1, i=0,1, \ldots, r-1, w_{0} \cdot \chi_{0}=\psi, w_{r} \cdot \chi_{0}=w \cdot \chi_{0}$ and the product

$$
\left(M\left(w_{r} \cdot \chi_{0}\right): L\left(w_{r-1} \cdot \chi_{0}\right)\right)\left(M\left(w_{r-1} \cdot \chi_{0}\right): L\left(w_{r-2} \cdot \chi_{0}\right)\right) \cdots\left(M\left(w_{1} \cdot \chi_{0}\right): L(\psi)\right)
$$

is nonzero.

Definition $5.3[12,17]$. A GCM $A$ is indecomposable if $A \neq\left[\begin{array}{ll}B & 0 \\ 0 & C\end{array}\right]$, where $B, C$ are GCM's.

Definition 5.4 [17]. An indecomposable symmetrizable GCM is said to be Euclidean if the associated symmetric bilinear form is positive semidefinite on the linear span of the roots.

We say that a system of roots $\Delta$ of a Kac-Moody Lie algebra $g$ is Euclidean (resp. classical) if $g$ comes from a Euclidean (resp. finite) GCM.

We make an additional assumption on $\chi$ :

(B) $\Delta_{\chi}$ is of rank 2, Euclidean or classical with associated bilinear form $(,)_{\chi}=$ ( , ) $)_{\mid R_{\chi}}$, where $R_{\chi}$ is the linear span of $\Delta_{\chi}$.

Let $W_{\chi}^{(i)}=\left\{w \in W_{\chi} \mid l_{\chi}(w)=i\right\}$. Then $W_{\chi}^{(i)}=\left\{w_{i}, w_{i}^{\prime}\right\}, w_{i}, w_{i}^{\prime}$ as in $\S 4, i \in \mathbf{Z}_{+}$.

THEOREM 5.5. Let $w \in W_{\chi, X}^{1} \cap W_{\chi}^{(k)}$. Then

$$
\operatorname{ch} L\left(w \cdot \chi_{0}\right)=\operatorname{ch} M\left(w \cdot \chi_{0}\right)+(-1)^{k} \sum_{\substack{i<k \\ w^{\prime} \in W_{x}^{(i)}}}(-1)^{i}\left(\operatorname{ch} M\left(w^{\prime} \cdot \chi_{0}\right)\right)
$$

Proof. Assume that $\left(\chi_{0}+\rho, \beta_{i}\right) \neq 0, i=1,2$. If $k=0$ then $w \cdot \chi=\chi_{0}$. If $\left(M\left(\chi_{0}\right): L(\nu)\right) \neq 0$ for some $\nu \in \mathfrak{h}^{*} \backslash\left\{\chi_{0}\right\}$ then we obtain a contradiction by Lemma 5.2. Hence $M\left(\chi_{0}\right)=L\left(\chi_{0}\right)$ and the theorem is true in this case. We now proceed by induction on $k$. We assume that the statement of the theorem holds for all $w \in W_{\chi}^{(i)}$ with $i<k$. Let $w \in W_{\chi}^{(k)}$. By Lemma 5.2 we may form for $w^{\prime} \in W_{\chi}^{(k-1)}$, and each $i \in \mathbf{Z}_{+}$the modules $\left(M\left(w \cdot \chi_{0}\right) / T\left(w \cdot \chi_{0}\right) M\left(w^{\prime} \cdot \chi_{0}\right)\right)_{i}$ as in $\S 3$. By Theorem 3.10, Lemma 5.2 and the induction hypothesis we obtain

$$
\sum_{i>0} \operatorname{ch}\left(M\left(w \cdot \chi_{0}\right) / T\left(w \cdot \chi_{0}\right) M\left(w^{\prime} \cdot \chi_{0}\right)\right)_{i}=\operatorname{ch} L\left(w_{k-1}^{\prime} \cdot \chi_{0}\right)
$$

(resp. ch $\left.L\left(w_{k-1} \cdot \chi_{0}\right)\right)$ if

$$
w^{\prime}=w_{k-1}
$$

(resp. if $w^{\prime}=w_{k-1}^{\prime}$ ). This implies that the unique maximal submodule of the highest weight module $M\left(w \cdot \chi_{0}\right) / T\left(w \cdot \chi_{0}\right) M\left(w^{\prime} \cdot \chi_{0}\right)$ is isomorphic with $L\left(w_{k-1}^{\prime} \cdot \chi_{0}\right)$ (resp. $\left.L\left(w_{k-1} \cdot \chi_{0}\right)\right)$ if $w^{\prime}=w_{k-1}\left(\right.$ resp. $\left.w^{\prime}=w_{k-1}^{\prime}\right)$. From this we obtain the result in this 
case. Suppose that $\left(\chi_{0}+\rho, \beta_{i}\right)=0$ for $i=1$ or $i=2$ and let $w$ be as in the assumption of the theorem. Then there is a unique $w^{\prime} \in W_{\chi, X}^{1} \cap W_{\chi}^{(k-1)}$. Proceeding as above we obtain $\sum_{i>0} \operatorname{ch}\left(M\left(w \cdot \chi_{0}\right) / T\left(w \cdot \chi_{0}\right) M\left(w^{\prime} \cdot \chi_{0}\right)\right)_{i}=0$. (See the proof of Lemma 5.10 for more details.) Hence $M\left(w \cdot \chi_{0}\right) / T\left(w \cdot \chi_{0}\right) M\left(w^{\prime} \cdot \chi_{0}\right)$ is irreducible. Therefore $\operatorname{ch} L\left(w \cdot \chi_{0}\right)=\operatorname{ch} M\left(w \cdot \chi_{0}\right)-\operatorname{ch} M\left(w^{\prime} \cdot \chi_{0}\right)$. This proves the theorem. Q.E.D.

REMARK. Theorem 5.5 proves the Kazhdan-Lusztig conjectures in our case.

It is well known that if $\Delta$ is classical and $\chi, \psi \in \mathfrak{h}^{*}$ then $(M(\chi): L(\psi)) \neq 0$ if and only if there are $\gamma_{1}, \ldots, \gamma_{k} \in \Delta_{+}$such that [1]

$$
\chi=\sigma_{\gamma_{k}} \cdots \sigma_{\gamma_{1}} \cdot \psi \geqslant \sigma_{\gamma_{k-1}} \cdots \sigma_{\gamma_{1}} \cdot \psi \geqslant \cdots \geqslant \psi \text {. }
$$

In this case we write $\psi \uparrow \chi$. Using this and Theorem 5.5 we obtain a result due to Jantzen [10].

COROllary 5.6 [10, SATZ 3.17]. let $\chi$ be an element of $\mathfrak{h}^{*}$ and let $\Delta_{\chi}$ be classical of rank 2. If $\psi \uparrow \chi$ then $(M(\chi): L(\psi))=1$.

Set $W_{\chi, X}^{1,(k)}=W_{\chi, X}^{1} \cap W_{\chi}^{(k)}$. By our assumptions (A) and (B) on $\chi, \# W_{\chi, X}^{1,(k)}=1$ or 2, all $k \in \mathbf{Z}_{+}$.

Corollary 5.7. Let $w \in W_{\chi}^{1,(k)}$. Then:

(i) $M\left(w \cdot \chi_{0}\right)_{i}=\Sigma_{w^{\prime}} M\left(w^{\prime} \cdot \chi_{0}\right)\left(w^{\prime} \in W_{\chi, X}^{1,(k-i)}\right), 0 \leqslant i \leqslant k$.

(ii) $M\left(w \cdot \chi_{0}\right)_{i} / M\left(w \cdot \chi_{0}\right)_{i+1}=\bigoplus_{w^{\prime}} L\left(w^{\prime} \cdot \chi_{0}\right)\left(w^{\prime} \in W_{\chi, X}^{1,(k-i)}\right), 0 \leqslant i \leqslant k$.

Proof. If $k=0$ then (i) and (ii) are vacuous. Let $k \geqslant 1$ and suppose that

$$
M\left(w \cdot \chi_{0}\right)_{1}=\sum_{w^{\prime}} M\left(w^{\prime} \cdot \chi_{0}\right) \quad\left(w^{\prime} \in W_{\chi, X}^{1,(j-1)}\right)
$$

for all $w \in W_{\chi, X}^{1,(j)}, 0 \leqslant j \leqslant k-1$. In particular,

$$
\bigcap_{w} M\left(w \cdot \chi_{0}\right)=\sum_{w^{\prime}} M\left(w^{\prime} \cdot \chi_{0}\right) \quad\left(w \in W_{\chi, X}^{1,(j)}, w^{\prime} \in W_{\chi, X}^{1,(j-1)}\right),
$$

$$
\text { for all } 0 \leqslant j \leqslant k-1 \text {. }
$$

This implies that

$$
\begin{array}{r}
(-1)^{k} \sum_{j<k-1}(-1)^{j} \sum_{w} \operatorname{ch} M\left(w \cdot \chi_{0}\right)=-\operatorname{ch} \sum_{w^{\prime}} M\left(w^{\prime} \cdot \chi_{0}\right) \\
\left(w \in W_{\chi, X}^{1,(j)}, w^{\prime} \in W_{\chi, X}^{1,(k-2)}\right) .
\end{array}
$$

By Theorem 5.5

$$
\begin{aligned}
\operatorname{ch} M\left(w \cdot \chi_{0}\right)_{1} & =\sum_{w^{\prime}} \operatorname{ch} M\left(w^{\prime} \cdot \chi_{0}\right)+(-1)^{k} \sum_{j<k-1}(-1)^{j} \sum_{w^{\prime \prime}} \operatorname{ch} M\left(w^{\prime \prime} \cdot \chi_{0}\right) \\
& =\sum_{w^{\prime}} \operatorname{ch} M\left(w^{\prime} \cdot \chi_{0}\right)-\operatorname{ch} \sum_{w^{\prime \prime \prime}} M\left(w^{\prime \prime \prime} \cdot \chi_{0}\right) \\
& =\sum_{w^{\prime}} \operatorname{ch} M\left(w^{\prime} \cdot \chi_{0}\right)-\operatorname{ch} \bigcap_{w^{\prime}} M\left(w^{\prime} \cdot \chi_{0}\right) \\
& =\operatorname{ch} \sum_{w^{\prime}} M\left(w^{\prime} \cdot \chi_{0}\right) \quad\left(w^{\prime} \in W_{\chi, X}^{1,(k-1)}, w^{\prime \prime} \in W_{\chi}^{1,(j)}, w^{\prime \prime \prime} \in W_{\chi}^{1,(k-2)}\right),
\end{aligned}
$$


for all $w \in W_{\chi, X}^{1,(k)}$. Now, $M\left(w \cdot \chi_{0}\right)_{1} \supset \Sigma_{w^{\prime}} M\left(w^{\prime} \cdot \chi_{0}\right)\left(w^{\prime} \in W_{\chi, X}^{1,(k-1)}\right)$ for all $w \in$ $W_{\chi, X}^{1,(k)}$, by Corollary 3.4. Hence $M\left(w \cdot \chi_{0}\right)_{1}=\Sigma_{w^{\prime}} M\left(w^{\prime} \cdot \chi_{0}\right)\left(w^{\prime} \in W_{\chi, X}^{1,(k-1)}\right)$, all $w \in W_{\chi, X}^{1,(k)}$, all $k \in \mathbf{Z}_{+}$. The corollary now follows using the argument of the proof of Theorem 4.16 with the following changes: instead of Proposition 4.7 and Lemma 4.11 we use the above, and instead of Corollary 4.14(i) we use Theorem 5.5. Q.E.D.

Using Theorem 5.5 and Corollary 5.7 we obtain

COROLlaRY 5.8. (i) If $X=\varnothing$, then given $w \in W_{\chi, X}^{1,(k)}=W_{\chi}^{(k)}$ there is a resolution of $L\left(w \cdot \chi_{0}\right)$ :

$$
\begin{aligned}
0 \rightarrow M\left(\chi_{0}\right) & \stackrel{\alpha_{k}}{\rightarrow} M\left(w_{1} \cdot \chi_{0}\right) \oplus M\left(w_{1}^{\prime} \cdot \chi_{0}\right) \stackrel{\alpha_{k-1}}{\rightarrow} \cdots \\
& \stackrel{\alpha_{2}}{\rightarrow} M\left(w_{k-1} \cdot \chi_{0}\right) \oplus M\left(w_{k-1}^{\prime} \cdot \chi_{0}\right) \stackrel{\alpha_{1}}{\rightarrow} M\left(w \cdot \chi_{0}\right) \stackrel{\varepsilon}{\rightarrow} L\left(w \cdot \chi_{0}\right) \rightarrow 0
\end{aligned}
$$

where $\alpha_{j}(x, y)=\left(i_{w_{k-j}, w_{k-j+1}} x+i_{w_{k-j}^{\prime}, w_{k-j+1}} y,-i_{w_{k-j}, w_{k-j+1}^{\prime}} x-i_{w_{k-j}^{\prime}, w_{k-j+1}^{\prime}} y\right), \quad 1 \leqslant j$ $\leqslant k, \alpha_{1}(x, y)=i_{w_{k-1}, w} x+i_{w_{k-1}^{\prime}, w} y$ and $i_{\tau, \tau^{\prime}}: M\left(\tau \cdot \chi_{0}\right) \rightarrow M\left(\tau^{\prime} \cdot \chi_{0}\right)$ are unique up to scalar embeddings, for $\tau \in W_{\chi}^{(i)}, \tau^{\prime} \in W_{\chi}^{(i+1)}$.

(ii) If $X \neq \varnothing$, then given $w \in W_{\chi, X}^{1,(k)}$ there is a resolution of $L\left(w \cdot \chi_{0}\right)$ :

$$
0 \rightarrow M\left(w^{\prime} \cdot \chi_{0}\right) \stackrel{\alpha_{1}}{\rightarrow} M\left(w \cdot \chi_{0}\right) \stackrel{\varepsilon}{\rightarrow} L\left(w \cdot \chi_{0}\right) \rightarrow 0
$$

where $W_{\chi, X}^{1,(k-1)}=\left\{w^{\prime}\right\}, \alpha_{1}$ the unique embedding up to scalar.

The following is also immediate from Theorem 5.5.

COROllary 5.9. Let $w, w^{\prime} \in W_{\chi, X}^{1,(k)}$. Then:

(i) $\left(M\left(w \cdot \chi_{0}\right): L\left(w^{\prime} \cdot \chi_{0}\right)\right)=1($ resp. 0$)$ if $w \leqslant_{\chi} w^{\prime}$ (resp. otherwise);

(ii) $\left(I\left(w^{\prime} \cdot \chi_{0}\right): M\left(w \cdot \chi_{0}\right)\right)=1($ resp. 0$)$ if $w \leqslant_{\chi} w^{\prime}$ (resp. otherwise).

REMARK. As in $\S 4$, Corollary 5.7(i) implies the heredity of Jantzen's filtration in our case: Let $w, w^{\prime} \in W_{\chi, X}^{1}$. If $l_{\chi}\left(w^{\prime}\right)-l_{\chi}(w)=r \in \mathbf{N}$ then $M\left(w \cdot \chi_{0}\right)_{i}=$ $M\left(w^{\prime} \cdot \chi_{0}\right)_{r+i}, 1 \leqslant i \leqslant l_{\chi}(w)$. Also, Corollary 5.8, Theorem 5.5, the statements (i) and (ii) of Corollary 5.7, the statements (i) and (ii) of Corollary 5.9 and the above statement on the heredity of Jantzen's filtration are all equivalent statements.

Next we will show that the above results almost complement the results of $\S 4$ in the case of rank 2 Euclidean root systems. First we need a general result. It will add the singular case left out in the analysis of $\S 4$. We keep the notation of $\S 4$. Let $X_{\mu}=\left\{\alpha_{i} \mid\left(\mu+\rho, \alpha_{i}\right)=0\right\}$. Let $W_{X_{\mu}}^{1}$ be defined as $W_{\chi, X}^{1}$ and set $W_{X_{\mu}}^{1,(k)}=W_{X_{\mu}}^{1} \cap$ $W^{(k)}$.

Lemma 5.10. Let $\mu+\rho \in P^{+}, w \in W_{X_{\mu}}^{1,(k)}$. Assume that $X_{\mu} \neq \Delta_{0}$. Then $\operatorname{ch} L(w \cdot \mu)$ $=\operatorname{ch} M(w \cdot \mu)+(-1)^{k} \sum_{i>k}(-1)^{i}\left(\operatorname{ch} M\left(w_{i} \cdot \mu\right)+\operatorname{ch} M\left(w_{i}^{\prime} \cdot \mu\right)\right)$.

Proof. The case where $\mu \in P^{+}$is contained in the proof of Corollary 4.13. Assume $X_{\mu} \neq \varnothing$. If $w=w_{k}$ (resp. $\left.w_{k}^{\prime}\right)$ we consider the modules $N\left(w_{k}, w_{k+1} ; \mu\right)_{i}=$ $\left(M\left(w_{k} \cdot \mu\right) / T\left(w_{k} \cdot \mu\right) M\left(w_{k+1} \cdot \mu\right)\right)_{i}\left(\right.$ resp. $\left.N\left(w_{k}^{\prime}, w_{k+1}^{\prime} ; \mu\right)_{i}\right)$ as before. Using Theorem 
3.10 and the diagram of $\S 4$ we obtain

$$
\begin{gathered}
\sum_{i>0} \operatorname{ch} N\left(w_{k}, w_{k+1} ; \mu\right)_{i}=\sum_{\substack{i>0 \\
i \text { odd }}}\left(\operatorname{ch} M\left(w_{k+i} \cdot \mu\right)+\operatorname{ch} M\left(w_{k+i}^{\prime} \cdot \mu\right)\right) \\
-\sum_{\substack{j>0 \\
j \text { even }}}\left(\operatorname{ch} M\left(w_{k+j} \cdot \mu\right)+\operatorname{ch} M\left(w_{k+j}^{\prime} \cdot \mu\right)\right)-\operatorname{ch} M\left(w_{k+1} \cdot \mu\right)
\end{gathered}
$$

(resp. $\quad \sum_{i>0} \operatorname{ch} N\left(w_{k}^{\prime}, w_{k+1}^{\prime} ; \mu\right)_{i}=\sum_{i>0, i \text { odd }}\left(\operatorname{ch} M\left(W_{k+i} \cdot \mu\right)+\operatorname{ch} M\left(w_{k+i}^{\prime} \cdot \mu\right)\right)-$ $\sum_{j>0, j \text { even }}\left(\operatorname{ch} M\left(w_{k+j} \cdot \mu\right)+\operatorname{ch} M\left(w_{k+j}^{\prime} \cdot \mu\right)\right)$ - ch $\left.M\left(w_{k+1}^{\prime} \cdot \mu\right)\right)$. Now, the sum on the right-hand side of the above equations telescopes to 0 . This implies that $N\left(w_{k}, w_{k+1} ; \mu\right)_{1}=0$ (resp. $\left.N\left(w_{k}^{\prime}, w_{k+1}^{\prime} ; \mu\right)_{1}=0\right)$, i.e. $N\left(w_{k}, w_{k+1} ; \mu\right)=L\left(w_{k} \cdot \mu\right)$ (resp. $\left.N\left(w_{k}^{\prime}, w_{k+1}^{\prime} ; \mu\right)=L\left(w_{k}^{\prime} \cdot \mu\right)\right)$. Therefore, $\operatorname{ch} L\left(w_{k} \cdot \mu\right)=\operatorname{ch} M\left(w_{k} \cdot \mu\right)-$ $\operatorname{ch} M\left(w_{k+1} \cdot \mu\right)$ (resp. $\left.\operatorname{ch} L\left(w_{k}^{\prime} \cdot \mu\right)=\operatorname{ch} M\left(w_{k}^{\prime} \cdot \mu\right)-\operatorname{ch} M\left(w_{k+1}^{\prime} \cdot \mu\right)\right)$. This proves the lemma. Q.E.D.

Next we prove a result for Euclidean GCM's of arbitrary rank. We denote by $\xi$ the principal null root as in [18].

LEMMA 5.11. Let $\Delta$ be Euclidean, $\xi \in \Delta$ as above, $\psi$ a real weight.

(i) If $(\psi+\rho, \xi)>0$ then there is $w \in W$ such that $(w(\psi+\rho))\left(h_{i}\right) \geqslant 0, i=1, \ldots, n$.

(ii) If $(\psi+\rho, \xi)<0$ then there is $w \in W$ such that $(w(\psi+\rho))\left(h_{i}\right) \leqslant 0, i=$ $1, \ldots, n$.

Proof. Assume $(\psi+\rho, \xi)<0$. Let $R_{+}(\psi)=\left\{\alpha \in \Delta_{+} \mid 2(\psi+\rho, \alpha)=c(\alpha, \alpha)\right.$, $c \in \mathbf{R}, c>0\}$. Then $R_{+}(\psi) \subset \Delta_{R}^{+}$. Now, $\Delta_{R}^{+}=\cup_{i=1}^{r}\left\{\gamma_{i}+p_{i} j \xi \mid j \in \mathbf{N}\right\}$ [19]. Therefore $\left|R_{+}(\psi)\right|$ is finite. If $R_{+}(\psi)=\varnothing$ then $\psi$ is negative. Suppose that there is $w \in W$ such that $w \cdot \nu$ is negative for all $\nu$ with $\left|R_{+}(\nu)\right|<\left|R_{+}(\psi)\right|$, where $\left|R_{+}(\psi)\right| \geqslant 1$. Let $\alpha \in R_{+}(\psi)$. Then there is $w \in W$ such that $w \sigma_{\alpha} \cdot \psi$ is negative by the induction assumption and since $\left|R_{+}\left(\sigma_{\alpha} \cdot \psi\right)\right|<\left|R_{+}(\psi)\right|$. This proves (ii). (i) is proved by replacing $\psi$ by $-\psi$ and using (ii). Q.E.D.

Combining Lemmas 5.10 and 5.11 with Theorems 5.5 and 4.12 we obtain the following.

THEOREM 5.12. Let $\Delta$ be Euclidean or classical and of rank 2. Let $\psi \in P$ and assume that $(\psi+\rho, \xi) \neq 0$ if $\Delta$ is Euclidean. Then there are $k \in \mathbf{N}, w \in W_{X_{\psi}^{1}(k)}$ such that:

(i) $\operatorname{ch} L(\psi)=\operatorname{ch} M(\psi)+(-1)^{k} \sum_{i<k}(-1)^{i}\left(\operatorname{ch} M\left(w_{i} \cdot \psi_{0}\right)+\operatorname{ch} M\left(w_{i}^{\prime} \cdot \psi_{0}\right)\right)$ if $(\psi+\rho, \xi)<0, \Delta$ Euclidean, where $\psi=w \cdot \psi_{0}, \psi_{0} \in \mathfrak{h}^{*}$.

(ii) $\operatorname{ch} L(\psi)=\operatorname{ch} M(\psi)+(-1)^{k} \sum_{i>k}(-1)^{i}\left(\operatorname{ch} M\left(w_{i} \cdot \phi_{0}\right)+\operatorname{ch} M\left(w_{i}^{\prime} \cdot \phi_{0}\right)\right)$ if $(\psi+\rho, \xi)>0, \Delta$ Euclidean, where $\psi=w \cdot \phi_{0}, \phi_{0} \in \mathfrak{h}^{*}$.

Remark. Theorem 5.12 proves the Kazhdan-Lusztig conjectures in the rank 2 Euclidean case for all $L(\psi)$ such that $(\psi+\rho, \xi) \neq 0$.

6. Resolutions and characters of highest weight modules over the Witt algebra. From here on, unless otherwise stated, $\mathfrak{g}$ will denote the Witt algebra, i.e., $\mathfrak{g}$ is the complex Lie algebra with basis $\left\{e_{i}\right\}_{i \in \mathbf{Z}}$, where

$$
\left[e_{i}, e_{j}\right]=(j-i) e_{i+j}, \quad i, j \in \mathbf{Z} .
$$

We set $\mathfrak{h}=\mathbf{C} e_{0}, \mathfrak{n}=\bigoplus_{i \in \mathbf{N}} \mathbf{C} e_{i}, \mathfrak{b}=\mathfrak{h} \oplus \mathfrak{n}$ and $\mathfrak{n}^{-}=\bigoplus_{i \in \mathbf{N}} \mathbf{C} e_{-i}$. 
Note. The subalgebra $\mathfrak{n}$ (resp. $\mathfrak{n}^{-}$) is generated by $\left\{e_{1}, e_{2}\right\}$ (resp. $\left\{e_{-1}, e_{-2}\right\}$ ).

The pair $(\mathfrak{g}, \mathfrak{h})$ has a grading as described in $\$ 1$ and obviously satisfies condition (T1) of $\S 1$. Proposition 6.2 shows in particular that condition (T2) of $\S 1$ is also satisfied.

Next we consider a variation of the pairing studied by Shapovalov. The following is contained in the proof of [7, Theorem 3.3].

Lemma 6.1 [7]. Let $\mathfrak{g}$ be a complex semisimple Lie algebra, $\mathfrak{h} \subset \mathfrak{g}$ a Cartan subalgebra and $\mathfrak{g}=\mathfrak{n}^{-} \oplus \mathfrak{h} \oplus \mathfrak{n}$ a triangular decomposition. Set $\mathfrak{b}=\mathfrak{h} \oplus \mathfrak{n}$ and $\mathfrak{b}^{-}=$ $\mathfrak{h} \oplus \mathfrak{n}^{-}$. If $\mu \in \mathfrak{h}^{*}$, let $\bar{M}(\mu)=U(\mathfrak{g}) \otimes_{U\left(\mathfrak{b}^{-}\right)} \mathbf{C}(\mu)$. Let $\phi$ be the projection of $U(\mathfrak{g})=$ $U(\mathfrak{h}) \oplus\left(\mathfrak{n}^{-} U(\mathfrak{g})+U(\mathfrak{g}) \mathfrak{n}\right)$ on the first factor. If $v_{\mu}=1 \otimes 1$ in $M(\mu), v_{-\mu}=1 \otimes 1$ in $\bar{M}(\mu)$ and $u, u^{\prime} \in U(g)$, we set $\left(u v_{\mu}, u v_{-\mu}\right)_{\mu}=-\mu\left(\phi\left(\sigma\left(u^{\prime}\right) u\right)\right)$, where $\sigma$ is the principal antiautomorphism of $U(\mathfrak{g})$. Then $(,)_{\mu}$ is a g-invariant pairing of $M(\mu)$ and $M(-\mu)$.

We set $H=2 e_{0}, X=-e_{1}$ and $Y=e_{-1}$. The Lie algebra $m$ generated by $\{Y, H, X\}$ is isomorphic with $\mathfrak{s} \mathfrak{l}(2, \mathbf{C})$. We set $\mathfrak{u}=\mathfrak{n} / \mathbf{C} e_{1}$ and $\mathfrak{u}^{-}=\mathfrak{n}^{-} / \mathbf{C} e_{-1}$.

Proposition 6.2. For each $r, s \in \mathbf{N}$, we set $\left(e_{r}, e_{-s}\right)=\delta_{r, s}\left(\begin{array}{c}r+1 \\ 3\end{array}\right)$ if $r, s \geqslant 2$, $\left(e_{r}, e_{-s}\right)=\delta_{r, s}$ otherwise. Then $($,$) extends to an \mathfrak{h}$-invariant pairing of $\mathfrak{n}$ and $\mathfrak{n}^{-}$ which induces an m-invariant pairing of $\mathfrak{u}$ and $\mathfrak{u}^{-}$.

Proof. $\mathfrak{u} \simeq U(\mathfrak{m}) \otimes_{U\left(\mathfrak{b}_{1}\right)} \mathbf{C}(4)$ and $\mathfrak{u}^{-} \simeq U(\mathfrak{m}) \otimes_{U\left(\mathfrak{b}_{1}^{-}\right)} \mathbf{C}(-4)$ as m-modules, where $\mathfrak{h}_{1}=\mathbf{C} H, \mathfrak{b}_{1}=\mathbf{C} H \oplus \mathbf{C} X, \mathfrak{b}_{1}^{-}=\mathbf{C} H \oplus \mathbf{C} Y$ and we identify $\mathfrak{h}_{1}^{*}$ with $\mathbf{C}$. We now apply Lemma 6.1 to $\mathfrak{m}, \mathfrak{h}_{1}, \mathfrak{b}_{1}, \mathfrak{b}_{1}^{-}$and $\mu \in \mathfrak{h}_{1}^{*}$ defined by $\mu(H)=4$. We use the notation of Lemma 6.1 and set $v_{\mu}=e_{2}, v_{-\mu}=e_{-2}$. Noting that $(\operatorname{ad} X)^{k} e_{2}=$ $(-1)^{k} k ! e_{2+k},(\operatorname{ad} Y)^{k} e_{-2}=(-1)^{k} k ! e_{-2-k}$ and $\left[X, Y^{k}\right]=k Y^{k-1}(H-k+1)$, for $k \in \mathbf{N}$, we obtain

$$
\begin{aligned}
\left(e_{2+k}, e_{-2-k}\right)_{\mu} & =\frac{1}{(k !)^{2}}\left(X^{k} v_{\mu}, Y^{k} v_{-\mu}\right)=\frac{(-1)^{k}}{(k !)^{2}}(-\mu)\left(\phi\left(X^{k} Y^{k}\right)\right) \\
& =\frac{(-1)^{k}}{(k !)^{2}} \prod_{j=1}^{k} k !(-j-3)=\frac{1}{k !} \prod_{j=1}^{k}(j+4) \\
& =\frac{1}{k !} k !\left(\begin{array}{c}
k+3 \\
3
\end{array}\right)=\left(\begin{array}{c}
k+3 \\
3
\end{array}\right)=\left(e_{2+k}, e_{-2-k}\right) .
\end{aligned}
$$

The proposition now follows from Lemma 6.1. Q.E.D.

RemarK. Proposition 6.2 shows that condition (S6) of [21] is satisfied for $(\mathfrak{g}, \mathfrak{h})$ and $S=\{1\}$.

We fix $(,)_{\mu}$ a nonzero contravariant form on $M(\mu)[10,11]$ and denote by $(,)_{\mu, m}$ its restriction to $M(\mu)_{\mu-m}, m \in \mathbf{Z}_{+}$.

TheOrem 6.3 [11, TheOREM 3]. Let $\mu \in$ C. Up to a nonzero constant

$$
\operatorname{det}(,)_{\mu, m}=\prod_{i=1}^{m}\left(\prod_{r \in \mathbf{N}, r \mid i}\left\{\mu+\frac{1}{24}\left[\left(3 r-2 \frac{i}{r}\right)^{2}-1\right]\right\}\right)^{p(m-i)}, \quad m \in \mathbf{N}
$$

where $p$ is the classical partition function. 
The nonnegative integers of the form $\frac{1}{2}\left(3 k^{2} \pm k\right)=\frac{1}{24}\left[(6 k \pm 1)^{2}-1\right]$, where $k \in \mathbf{Z}_{+}$, are called Euler's pentagonal numbers. We let $s_{k}$ (resp. $t_{k}$ ) be the nonnegative integer $\frac{1}{2}\left(3 k^{2}+k\right)$ (resp. $\left.\frac{1}{2}\left(3 k^{2}-k\right)\right), k \in \mathbf{Z}_{+}$, and let $P=\left\{s_{k}, t_{k} \mid k\right.$ $\left.\in \mathbf{Z}_{+}\right\}$.

Proposition 6.4. Let $\nu \in \mathrm{C}$. There is a filtration of submodules $M(\nu)=M(\nu)_{0} \supset$ $M(\nu)_{1} \supset \cdots$ such that:

(i) $M(\nu)_{1}$ is the largest proper submodule of $M(\nu)$;

(ii) for every $i \in \mathbf{N}$, there is on $M(\nu)_{i} / M(\nu)_{i+1}$ a nondegenerate contravariant form $(,)_{i} ;$ and

(iii) if $k \in \mathbf{Z}_{+}$and $\nu=s_{k}$ or $t_{k}$ then the following formula holds:

$$
\sum_{i>0} \operatorname{ch} M(-v)_{i}=\sum_{l \in \mathbf{N}, l \text { odd }} \operatorname{ch} M\left(-s_{k+l}\right)+\operatorname{ch} M\left(-t_{k+l}\right) .
$$

Proof. The construction of the filtration and the proofs of (i) and (ii) are as in Proposition 4.4. Now, $\operatorname{det}(,)_{-\nu, m}=0$ if and only if there are $r, i \in \mathbf{N}$ with $r \mid i$ and $1 \leqslant i \leqslant m$ such that $\frac{1}{24}\left[(6 k \pm 1)^{2}-1\right]=\frac{1}{24}\left[(3 r-2 i / r)^{2}-1\right]$, that is, such that $(6 k \pm 1)^{2}=(3 r-2 i / r)^{2}$. On one hand $6 k \pm 1=3 r-2 i / r$ with $r \mid i$ if and only if $i=\frac{1}{2}\left(3 r^{2}-6 k r \mp r\right)$ with $r \mid i$, that is, $i=\frac{1}{2}\left(3 r^{2}-6 k r \mp r\right)$ with $r \in \mathbf{N}, r$ odd. On the other hand, $6 k \pm 1=-3 r+2 i / r$ with $r \mid i$ if and only if $i=\frac{1}{2}\left(3 r^{2}+6 k r \pm r\right)$ with $r \in \mathbf{N}, r$ odd. Hence we have respectively

$$
-\frac{1}{2}\left(3 k^{2} \pm k\right)-i=-\frac{1}{2}\left[3(k-r)^{2} \pm(k-r)\right]
$$

and

$$
-\frac{1}{2}\left(3 k^{2} \pm k\right)-i=-\frac{1}{2}\left[3(k+r)^{2} \pm(k+r)\right]
$$

with $r \in \mathbf{N}, r$ odd. Proceeding as in the proof of Proposition 4.4(iii) we obtain

$$
\sum_{i>0} \operatorname{ch} M\left(-\frac{1}{2}\left(3 k^{2} \pm k\right)\right)_{i}=\sum \operatorname{ch} M\left(-\frac{1}{2}\left[3(k+r)^{2} \pm(k+r)\right]\right)
$$

where the sum on the right-hand side is over all $r \in \mathbf{N}, r$ odd. (6.1) now follows. Q.E.D.

COROllary 6.5. Let $k \in \mathbf{Z}_{+}$and $\nu=s_{k}$ or $t_{k}$. Then $\left(M(-\nu): L\left(-s_{k+1}\right)\right)=$ $\left(M(-\nu): L\left(-t_{k+1}\right)\right)=1$.

Proof. $\Sigma_{i>0}\left(M(-v)_{i}: L\left(-s_{k+1}\right)\right)=\Sigma_{i>0}\left(M(-\nu)_{i}: L\left(-t_{k+1}\right)\right)=1$, by (6.1). The result is now obvious. Q.E.D.

By the above, if $k \in \mathbf{Z}_{+}$and $\nu=-s_{k}$ or $\nu=-t_{k}$ then

$\operatorname{dim} \operatorname{Hom}_{\mathfrak{g}}\left(M\left(-s_{k+1}\right), M(\nu)\right) \leqslant 1$ and $\operatorname{dim}_{\operatorname{Hom}_{\mathfrak{g}}}\left(M\left(-t_{k}\right), M(\nu)\right) \leqslant 1$.

In [5 and 6] Goncharova computed the homology and cohomology of $\mathfrak{n}^{-}$with trivial coefficients. We state her theorem in our notation.

TheOREM 6.6 [5, 6]. Let $k \in \mathbf{Z}_{+}$and $\mathbf{C}$ denote the trivial g-module. Then $\left(H_{k}\left(\mathfrak{n}^{-}, \mathbf{C}\right)\right)_{s_{k}}=\mathbf{C}\left(-s_{k}\right),\left(H_{k}\left(\mathfrak{n}^{-}, \mathbf{C}\right)\right)_{-t_{k}}=\mathbf{C}\left(-t_{k}\right)$ and $\left(H_{k}\left(\mathfrak{n}^{-}, \mathbf{C}\right)\right)_{\mu}=(0)$ if $\mu \notin$ $\left\{-s_{k},-t_{k}\right\}$. (Here $\mathbf{C}(\mu)$ denotes the one-dimensional $\mathfrak{h}$-module where $e_{0}$ acts as $\mu$.) 
Using the above homology computation, Theorem 6.3 and our results of $\$ 1$ we obtain

THEOREM 6.7. (i) For each $k \geqslant 1$ there exist $\mathrm{g}$-module embeddings $\gamma_{k}: M\left(-s_{k}\right) \rightarrow$ $M(0), \delta_{k}: M\left(-t_{k}\right) \rightarrow M(0)$ such that $\operatorname{im} \gamma_{k}+\operatorname{im} \delta_{k}=\operatorname{im} \gamma_{k-1} \cap \operatorname{im} \delta_{k-1}, k \geqslant 2$.

(ii) There exists a resolution of the trivial $\mathrm{g}$-module $\mathrm{C}$ :

$$
\cdots \rightarrow M\left(-s_{k}\right) \oplus M\left(-t_{k}\right) \stackrel{\alpha_{k}}{\rightarrow} \cdots \stackrel{\alpha_{2}}{\rightarrow} M\left(-s_{1}\right) \oplus M\left(-t_{1}\right) \stackrel{\alpha_{1}}{\rightarrow} M(0) \stackrel{\varepsilon}{\rightarrow} \mathrm{C} \rightarrow 0
$$

where $\alpha_{k}(x, y)=(x+y,-(x+y)), k \geqslant 2, \alpha_{1}(x, y)=x+y$ and $\varepsilon$ is the canonical surjective homomorphism. (Here, we have identified $M\left(-s_{k}\right), M\left(-t_{k}\right)$ with their images under $\gamma_{k}$ and $\delta_{k}$ in $M(0)$, for simplicity.)

Proof. We prove (i) and (ii) simultaneously by induction on $k$. Let $E_{1}$ denote the unique maximal submodule of $M(0)$. Then we have a short exact sequence

$$
0 \rightarrow E_{1} \stackrel{\varepsilon_{1}}{\rightarrow} M(0) \stackrel{\varepsilon}{\rightarrow} \mathrm{C} \rightarrow 0 .
$$

By the long exact Tor-sequence in the first variable we obtain an exact sequence

$$
0 \rightarrow H_{1}\left(\mathfrak{n}^{-}, \mathrm{C}\right) \rightarrow H_{0}\left(\mathfrak{n}^{-}, E_{1}\right) \rightarrow 0 .
$$

Hence $H_{0}\left(\mathrm{n}^{-}, E_{1}\right) \simeq H_{1}\left(\mathfrak{n}^{-}, \mathbf{C}\right)=\mathbf{C}\left(-s_{1}\right) \oplus \mathbf{C}\left(-t_{1}\right)$ by Theorem 6.6. By Corollary 1.7 and Theorem $6.3 E_{1}$ is generated by $n$-invariant vectors of weights $-s_{1}$ and $-t_{1}$. Let $\gamma_{1}$ and $\delta_{1}$ be the compositions of the corresponding embeddings with $\varepsilon_{1}$. We identify $M\left(-s_{1}\right)$ and $M\left(-t_{1}\right)$ with their images, for simplicity. Let $\alpha_{1}: M\left(-s_{1}\right) \oplus$ $M\left(-t_{1}\right) \rightarrow M(0)$ be defined by $\alpha_{1}(x, y)=x+y$. Then we have

$$
M\left(-s_{1}\right) \oplus M\left(-t_{1}\right) \stackrel{\alpha_{1}}{\rightarrow} M(0) \stackrel{\varepsilon}{\rightarrow} \mathrm{C} \rightarrow 0
$$

with $\operatorname{im} \alpha_{1}=\operatorname{ker} \varepsilon$. Furthermore we have a short exact sequence

$$
0 \rightarrow E_{2} \stackrel{\varepsilon_{2}}{\rightarrow} M\left(-s_{1}\right) \oplus M\left(-t_{1}\right) \stackrel{\beta_{1}}{\rightarrow} E_{1} \rightarrow 0
$$

where $\varepsilon_{1} \circ \beta_{1}=\alpha_{1}$. Then we obtain, as before, exact sequences

$$
\begin{aligned}
& 0 \rightarrow H_{1}\left(\mathfrak{n}^{-}, E_{1}\right) \rightarrow H_{0}\left(\mathfrak{n}^{-}, E_{2}\right) \rightarrow 0, \\
& 0 \rightarrow H_{2}\left(\mathfrak{n}^{-}, \mathbf{C}\right) \rightarrow H_{1}\left(\mathfrak{n}^{-}, E_{1}\right) \rightarrow 0 .
\end{aligned}
$$

Hence $H_{0}\left(\mathfrak{n}^{-}, E_{2}\right) \simeq H_{1}\left(\mathfrak{n}^{-}, E_{1}\right) \simeq H_{2}\left(\mathfrak{n}^{-}, \mathbf{C}\right)=\mathbf{C}\left(-s_{2}\right) \oplus \mathbf{C}\left(-t_{2}\right)$ by Theorem 6.6. By Proposition 1.7 and Theorem $6.3 E_{2}$ is generated by n-invariant vectors of weights $-s_{2}$ and $-t_{2}$. Let $u=\left(u_{1}, u_{2}\right): M\left(-s_{2}\right) \rightarrow M\left(-s_{1}\right) \oplus M\left(-t_{1}\right)$ and $v=$ $\left(v_{1}, v_{2}\right): M\left(-t_{2}\right) \rightarrow M\left(-s_{1}\right) \oplus M\left(-t_{1}\right)$ be the compositions of the corresponding embeddings with $\varepsilon_{2}$. By exactness $u_{1}(x)=-u_{2}(x)$ and $v_{1}(y)=-v_{2}(y)$ in $M(0)$. Let $\gamma_{2}=u_{1}$ and $\delta_{2}=v_{1}$. We identify $M\left(-s_{2}\right)$ and $M\left(-t_{2}\right)$ with their images in $M(0)$. Then $M\left(-s_{2}\right)+M\left(-t_{2}\right)=M\left(-s_{1}\right) \cap M\left(-t_{1}\right)$. Let $\alpha_{2}: M\left(-s_{2}\right) \oplus M\left(-t_{2}\right)$ $\rightarrow M\left(-s_{1}\right) \oplus M\left(-t_{1}\right)$ be defined by $\alpha_{2}(x, y)=(x+y,-(x+y))$. Suppose we have defined $\gamma_{j}, \delta_{j}, 2 \leqslant j \leqslant k-1$, such that $\operatorname{im} \gamma_{j}+\operatorname{im} \delta_{j}=\operatorname{im} \gamma_{j-1} \cap \operatorname{im} \delta_{j-1}$, and 
we have identified $M\left(-s_{j}\right)$ and $M\left(-t_{j}\right)$ with their images in $M(0)$. Suppose, in addition, that we have

$$
\begin{aligned}
M\left(-s_{k-1}\right) \oplus M\left(-t_{k-1}\right) & \stackrel{\alpha_{k-1}}{\rightarrow} M\left(-s_{k-2}\right) \oplus M\left(-t_{k-2}\right) \\
& \rightarrow \cdots \rightarrow M\left(-s_{1}\right) \oplus M\left(-t_{1}\right) \stackrel{\alpha_{1}}{\rightarrow} M(0) \stackrel{\varepsilon}{\rightarrow} \mathrm{C} \rightarrow 0
\end{aligned}
$$

where $\alpha_{j}(x, y)=(x+y,-(x+y))$, im $\alpha_{j}=\operatorname{ker} \alpha_{j-1}$, and an exact sequence

$$
0 \rightarrow E_{j+1} \stackrel{\varepsilon_{j+1}}{\rightarrow} M\left(-s_{j}\right) \oplus M\left(-t_{j}\right) \stackrel{\beta_{j}}{\rightarrow} E_{j} \rightarrow 0
$$

where $H_{0}\left(\mathfrak{n}^{-}, E_{j}\right) \simeq \mathbf{C}\left(-s_{j}\right) \oplus \mathbf{C}\left(-t_{j}\right)$ and $\varepsilon_{j} \circ \beta_{j}=\alpha_{j}, 2 \leqslant j \leqslant k-1$. By induction we obtain $H_{j}\left(\mathfrak{n}^{-}, E_{k-j}\right) \simeq H_{j-1}\left(\mathfrak{n}^{-}, E_{k-j+1}\right)$ for $1 \leqslant j \leqslant k-1$, and $H_{k}\left(\mathrm{n}^{-}, \mathrm{C}\right) \simeq H_{k-1}\left(\mathfrak{n}^{-}, E_{1}\right)$. Now $\gamma_{k}, \delta_{k}$ and $\alpha_{k}$ are defined in the same way as $\gamma_{2}, \delta_{2}$ and $\alpha_{2}$ and the same argument for $k=2$ completes the induction step. Q.E.D.

Scholium 6.8. Let $k \in \mathbf{Z}_{+}, \chi \in\left\{s_{k}, t_{k}\right\}$ and $\psi \in\left\{s_{k+1}, t_{k+1}\right\}$. Then $\operatorname{Hom}_{\mathfrak{g}}(M(-\psi), M(-\chi))=\mathbf{C}$.

Definition 6.9. Let $\nu \in\left\{s_{k}, t_{k}\right\}, k \in \mathbf{Z}_{+}$. We define $M(-\nu)^{i}=M\left(-s_{k+i}\right)+$ $M\left(-t_{k+i}\right), i \in \mathrm{N}$, using the identifications in Theorem 6.7(ii).

Proposition 6.10. $M\left(-s_{k}\right)+M\left(-t_{k}\right)=M\left(-s_{k-1}\right) \cap M\left(-t_{k-1}\right), k \in \mathbf{N}$. If $\nu \in$ $\left\{s_{k}, t_{k}\right\}$ then

$$
M(-\nu) \supset M(-\nu)^{1} \supset M(-\nu)^{2} \supset \cdots
$$

is a $\mathrm{g}$-module filtration and

$$
\sum_{i \geqslant 1} \operatorname{ch} M(-\nu)^{i}=\sum_{l \in \mathbf{N}, l \text { odd }} \operatorname{ch} M\left(-s_{k+l}\right)+\operatorname{ch} M\left(-t_{k+l}\right)
$$

Proof. The first assertion is just a restatement of Theorem 6.7(i). (6.3) is proved in the same way as Proposition 4.7, using Theorem 6.7(i), (ii) rather than Theorem 2.4. Q.E.D.

Definition 6.11. Let $\nu \in P$. We define $\tilde{L}(-\nu)=M(-\nu) / M(-\nu)^{1}$.

Proposition 6.12. Let $\nu \in\left\{s_{k}, t_{k}\right\}, k \in \mathbf{Z}_{+}$. There exists a resolution of $\tilde{L}(-\nu)$

$$
\begin{aligned}
\cdots \rightarrow M\left(-s_{i}\right) \oplus M\left(-t_{i}\right) \stackrel{\alpha_{i}}{\rightarrow} \ldots \stackrel{\alpha_{k+2}}{\rightarrow} M\left(-s_{k+1}\right) \oplus M\left(-t_{k+1}\right) \\
\stackrel{\eta_{k+1}}{\rightarrow} M(-\nu) \stackrel{\varepsilon_{k}}{\rightarrow} \tilde{L}(-\nu) \rightarrow 0
\end{aligned}
$$

where the $\alpha_{i}, i \geqslant k+2$, are defined as in Theorem 6.7, $\eta_{k+1}(x, y)=x+y$ and $\varepsilon_{k}$ is the canonical projection.

Proof. We use the same argument as the one used in the proof of Proposition 4.9, where we replace Proposition 4.7 by Proposition 6.10 .

LeMmA 6.13. Let $\nu \in\left\{s_{k}, t_{k}\right\}, k \in \mathbf{Z}_{+}$. If $M(-\nu)_{j}=M(-\nu)^{j}$ for $1 \leqslant j \leqslant i$ then

$$
M(-\nu)_{i} / M(-\nu)_{i+1}=L\left(-s_{k+i}\right) \oplus L\left(-t_{k+i}\right) .
$$


Proof. The proof is identical to that of Lemma 4.15, where we use (6.1) instead of (4.2) and Proposition 6.10 instead of Proposition 4.7. Q.E.D.

TheOREM 6.14. Let $\nu \in\left\{s_{k}, t_{k}\right\}, \nu^{\prime} \in\left\{s_{j}, t_{j}\right\}, k, j \in \mathbf{Z}_{+}$. Then the following statements are equivalent.

(i) $\left(M(-\nu): L\left(-\nu^{\prime}\right)\right)=1\left(\right.$ resp. 0) if $k<j$ or $\nu=\nu^{\prime}$ (resp. otherwise).

(ii) $\left(I\left(-\nu^{\prime}\right): M(-\nu)\right)=1$ (resp. 0$)$ if $k<j$ or $\nu=\nu^{\prime}$ (resp. otherwise).

(iii) $\tilde{L}(-\nu)$ is irreducible for all $k \in \mathbf{Z}_{+}$.

(iv) $M(-\nu)^{i}=M(-\nu)_{i}$ for all $k \in \mathbf{Z}_{+}, i \in \mathbf{N}$.

(v) $M(-\nu)^{i} \subset M(-\nu)_{i}$ for all $k \in \mathbf{Z}_{+}, i \in \mathbf{N}$.

(vi) If $j-k=r \in \mathbf{N}$ then $M(-\nu)_{i}=M\left(-\nu^{\prime}\right)_{r+i}$ for all $i \in \mathbf{N}$.

(vii) $M(-\nu)_{i} / M(-\nu)_{i+1}=L\left(-s_{k+i}\right) \oplus L\left(-t_{k+i}\right)$ for all $k \in \mathbf{Z}_{+}, i \in \mathbf{N}$.

(viii) There exists a resolution of $L(-\nu)$

$$
\begin{aligned}
\cdots \rightarrow M\left(-s_{i}\right) \oplus M\left(-t_{i}\right) \stackrel{\alpha_{i}}{\rightarrow} \cdots \stackrel{\alpha_{k+2}}{\rightarrow} M\left(-s_{k+1}\right) \oplus M\left(-t_{k+1}\right) \\
\stackrel{\eta_{k+1}}{\rightarrow} M(-v) \stackrel{\varepsilon_{k}}{\rightarrow} L(-\nu) \rightarrow 0
\end{aligned}
$$

with $\alpha_{i}, i \geqslant k+2, \eta_{k+1}$ and $\varepsilon_{k}$ as in Proposition 6.12.

(ix) $\operatorname{ch} L(-\nu)=\operatorname{ch} M(-\nu)+(-1)^{k} \sum_{i>k}(-1)^{i}\left(\operatorname{ch} M\left(-s_{i}\right)+\operatorname{ch} M\left(-t_{i}\right)\right)$.

(x) $\operatorname{ch} M(-\nu)=\operatorname{ch} L(-\nu)+\sum_{i \in \mathrm{N}}\left(\operatorname{ch} L\left(-s_{k+i}\right)+\operatorname{ch} L\left(-t_{k+i}\right)\right)$.

Proof. We use the same arguments used in $\$ 4$ with the following modifications: Instead of Proposition 4.7, Proposition 4.9, Theorem 2.3 and Diagram 4, and (4.2) we use Proposition 6.10, Proposition 6.12, Theorem 6.7, and (6.1), respectively. Q.E.D.

REMARK. In [22] we prove 6.14(iii) and hence all of the parts of Theorem 6.14.

\section{REFERENCES}

1. I. N. Bernstein, I. M. Gelfand and S. I. Gelfand, Structure of representations generated by vectors of highest weight, Funkcional. Anal. i Priložen. (1) 5 (1971), 1-9.

2. N. Bourbaki, Groupes et algèbres de Lie, Eléments de Mathématique, Chap. IV-VI, Hermann, Paris, 1968.

3. J. Dixmier, Enveloping algebras, North-Holland Math. Library, no. 14, North-Holland, New York, 1977.

4. H. Garland and J. Lepowsky, Lie algebra homology and the Macdonald-Kac formulas, Invent. Math. 34 (1976), 37-76.

5. L. V. Goncharova, The cohomologies of Lie algebras of formal vector fields on the line, Funkcional. Anal. i Priložen. (2) 7 (1973), 6-14.

6. Cohomologies of Lie algebras of formal vector fields on the straight line, Funkcional. Anal. $\mathrm{i}$ Priložen. (3) 7 (1973), 33-44.

7. R. Goodman and N. R. Wallach, Whittaker vectors and conical vectors, J. Funct. Anal. (2) 39 (1980), 199-277.

8. P. J. Hilton and U. Stammbach, A course in homological algebra, Springer-Verlag, Berlin and New York, 1971.

9. J. C. Jantzen, Kontravariante Formen auf induzierten Darstellungen halbeinfacher Lie-Algebren, Math. Ann. 226 (1977), 53-65.

10. __ Moduln mit einem höchsten Gewicht, Lecture Notes in Math., vol. 750, Springer-Verlag, Berlin and New York, 1979.

11. V. G. Kac, Contravariant form for Lie algebras and superalgebras, Lecture Notes in Physics, vol. 94, Springer-Verlag, Berlin and New York, 1979, pp. 441-445. 
12. __ Simple irreducible graded Lie algebras of finite growth, Math. USSR Izv. 2 (1968), 1271, 1311.

13. , Some problems on infinite dimensional Lie algebras and their representations (for AMS meeting at Amherst, 1981), preprint.

14. V. G. Kac and D. A. Kazhdan, Structure of representations with highest weight of infinite dimensional Lie algebras, Adv. in Math. 34 (1979), 97-108.

15. D. Kazhdan and G. Lusztig, Representations of coxeter groups and Hecke algebras, Invent. Math. 53 (1979), 165-184.

16. J. Lepowsky, Lectures on Kac-Moody Lie algebras, Université de Paris VI (1978) (mimeographed notes).

17. R. V. Moody, A new class of Lie algebras, J. Algebra 10 (1968), 211-230.

18. ___ Euclidean Lie algebras, Canad. J. Math. 21 (1969), 1432-1454.

19. Root systems of hyperbolic type, Adv. in Math. 33 (1979), 144-160.

20. A. Rocha-Caridi, Resolutions of irreducible highest weight modules over infinite dimensional graded Lie algebras, Proc. 1981 Conf. on Lie Algebras and Related Topics, Lecture Notes in Math., vol. 933, Springer-Verlag, Berlin and New York, 1982.

21. A. Rocha-Caridi and N. R. Wallach, Projective modules over graded Lie algebras. I, Math. Z. 180 (1982), 151-177.

22.

, Characters of irreducible representations of the Lie algebra of vector fields on the circle, Invent. Math. (to appear).

23. N. N. Shapovalov, On a bilinear form on the universal enveloping algebra of a complex semi-simple Lie algebra, Funct. Anal. Appl. 6 (1972), 307-312.

School of Mathematics, Institute for Advanced Study, Princeton, New Jersey 08540

Center for Mathematical Sciences Research, Rutgers University, New Brunswick, New JERSEY 08903 